\title{
Review
}

Yuhua Chang, Jingxuan Wei and Chengkuo Lee*

\section{Metamaterials - from fundamentals and MEMS tuning mechanisms to applications}

https://doi.org/10.1515/nanoph-2020-0045

Received January 21, 2020; accepted April 1, 2020; published online May 14, 2020

Abstract: Metamaterials, consisting of subwavelength resonant structures, can be artificially engineered to yield desired response to electromagnetic waves. In contrast to the naturally existing materials whose properties are limited by their chemical compositions and structures, the optical response of metamaterials is controlled by the geometrics of resonant unit cells, called "meta-atoms". Many exotic functionalities such as negative refractive index, cloaking, perfect absorber, have been realized in metamaterials. One recent technical advance in this field is the active metamaterial, in which the structure of metamaterials can be tuned to realize multiple states in a single device. Microelectromechanical systems (MEMS) technology, well-known for its ability of reconfiguring mechanical structures, complementary metal-oxide-semiconductor (CMOS) compatibility and low power consumption, is perfectly suitable for such purpose. In the past one decade, we have seen numerous exciting works endeavoring to incorporate the novel MEMS functionalities with metamaterials for widespread applications. In this review, we will first visit the fundamental theories of MEMS-based active metamaterials, such as the lumped circuit model, coupled-mode theory, and interference theory. Then, we summarize the recent applications of MEMS-based metamaterials in various research fields. Finally, we provide an

*Corresponding author: Chengkuo Lee, Department of Electrical and Computer Engineering, National University of Singapore, Singapore, 117583, Singapore; Center for Intelligent Sensors and MEMS, National University of Singapore, Singapore, 117608, Singapore; and National University of Singapore Suzhou Research Institute (NUSRI), Suzhou, 215123, PR China, E-mail: elelc@nus.edu.sg. https://orcid.org/00000002-8886-3649

Yuhua Chang and Jingxuan Wei: Department of Electrical and Computer Engineering, National University of Singapore, Singapore, 117583, Singapore; Center for Intelligent Sensors and MEMS, National University of Singapore, Singapore, 117608, Singapore; and National University of Singapore Suzhou Research Institute (NUSRI), Suzhou, 215123, PR China outlook on the future research directions of MEMS-based metamaterials and their possible applications.

Keywords: microelectromechanical system (MEMS); plasmonically enhanced physical sensor; radiation detector; reconfigurable metamaterial.

\section{Introduction}

Metamaterials, which consist of subwavelength resonant structures, have garnered significant research attention since the first experimental demonstration of negative refractive index [1]. One of the unprecedented features in such metamaterials is that their effective properties can be artificially engineered, sharply different from the traditional naturally existing materials whose properties are limited by the chemical compositions. Thanks to the design flexibility offered by metamaterials, numerous intriguing phenomena have been explored and investigated with applications in a broad frequency range, such as perfect absorption [2-5], slow light [6-8], metalens [9-11] and chiral metamaterials [12-14]. As one of the recent advanced technologies, active metamaterials hold the promise for the dynamic modulation of light, including amplitude, phase, beam shape and etc. To implement the active control of electromagnetic (EM) wave, many materials have been reported in the past a few years, such as liquid crystals $[15$, 16], graphene [17-19], phase change materials [20-22], ferroelectric materials $[23,24]$ and semiconductors $[25,26]$. These materials provide various tuning mechanisms. Liquid crystals can orient itself from the anisotropic state into the isotropic state electrically [27, 28], optically [29, 30], magnetically [31] and thermally [32], creating change in the optical properties such as the refractive index. Liquid crystal can be employed as either the ambient background or the metasurface itself. Graphene has a low carrier density of states near the Dirac point so that the gate voltage can tune the Fermi level [33], as well as the resonance frequency. The graphene itself can also be patterned as resonators [34]. Ferroelectric materials such as lithium niobate $\left(\mathrm{LiNbO}_{3}\right)$ and barium strontium titanate (BST) can 
tune the refractive index thermally and electrically a result of their asymmetry crystal structure. The contrast in the refractive index is more significant if ferroelectric materials are operated around the Curie temperature. Two types of phase change materials are recently emerging: chalcogenide and vanadium dioxide $\left(\mathrm{VO}_{2}\right)$. Chalcogenide phase change material is a ternary compound consisting of germanium (Ge), antimony (Sb), and tellurium (Te). It can transit between the crystal state and amorphous state upon the heating and cooling cycle and both of these two states are nonvolatile with zero holding power [21, 22]. $\mathrm{VO}_{2}$, on the other hand, can transit between metal and insulator state upon heating $[35,36]$. Semiconductor, which can be either the substrate or part of the metamaterials, can photoexcite the extra carriers by laser and thus tune the electric properties of the resonance.

Among these tuning mechanisms, the most straightforward and efficient method is to geometrically change the configurations of the unit cells because the effective properties of metamaterials are determined by these unit cells. This reconfiguration is enabled by the mature actuating schemes in microelectromechanical system (MEMS) technology, which can induce the displacement inside or between the unit cells [37]. MEMS actuators provide general tuning methods that can be applied to a broad frequency range from terahertz (THz) to mid-infrared (MIR) and even visible light. Although the shrinking feature sizes of the metamaterials requires the reduced dimension of actuators and more advanced lithography techniques, the overall tuning mechanisms remain unchanged. Besides, the monolithic integration of the MEMS actuators and metamaterials are compatible with the current Complementary-Metal-Oxide-Semiconductor (CMOS) fabrication platform.

MEMS technology contains not only the actuators as tuning methods in metamaterials but also various physical sensors that transform stimulus signals from the ambient environment into the electrical domains. In particular, the most promising physical sensor that can be integrated with metamaterials is the MEMS radiation detector in MIR and $\mathrm{THz}$ where the incident EM wave is absorbed and transduced into heat. Because the conventional radiation detectors like thermopiles and bolometers lack wavelength selectivity and high absorption efficiency $[38,130]$, the incorporation of metamaterials helps to overcome these two bottlenecks.

In this review, we start from a brief overview of recent advances in the MEMS-based metamaterials, followed by their fundamentals that include lumped equivalent circuit model, temporal coupled-mode theory, interference theory and MEMS actuating mechanisms. In Section 3, we will introduce the MEMS actuated metamaterials, which can be categorized into the modulation of EM waves, manipulation of the wavefront, tunable metasurface-based holograms, tunable emitters/ perfect absorbers and logic operations. In Section 4, we will focus on the plasmonically enhanced physical sensors, including radiation sensors, strain sensors, spectrometer and etc. Finally, we will give an outlook and discussion about future technologies in this field.

\section{Metamaterials and MEMS}

A large portion of MEMS technologies can be classified into two categories: actuators and sensors, which are collectively known as transducers. Actuators transform nonmechanical energy into mechanical motion, force or torque. On the contrary, sensors usually transform mechanical motion, which is the result of ambient environment, into electric or optical outputs. Therefore, the combination of MEMS and metamaterials creates two applications as shown in Figure 1: MEMS actuated metamaterials and plasmonically enhanced physical sensors. In the MEMS actuated metamaterials, actuators enable the structure reconfiguration and the active control of the unit cell,

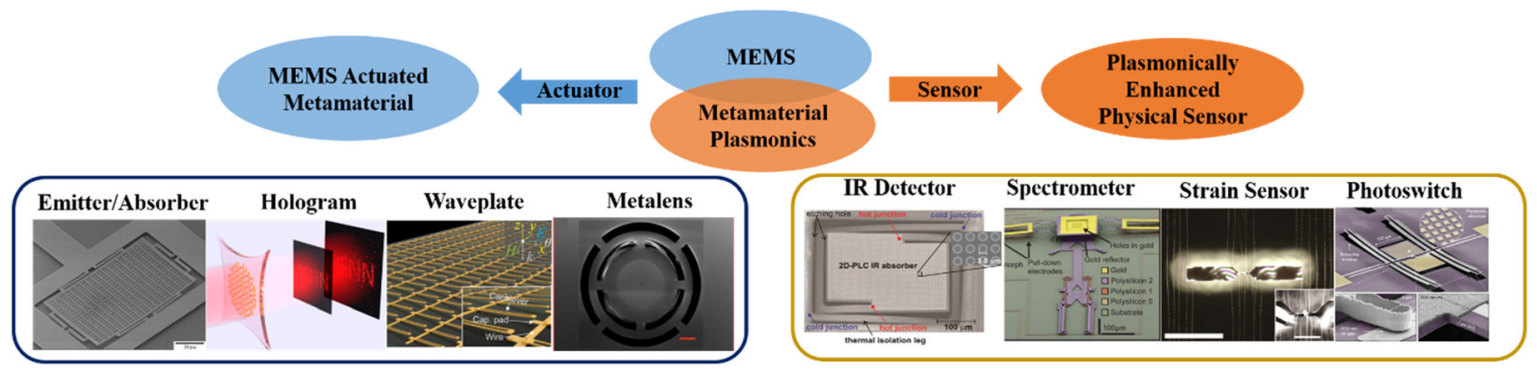

Figure 1: A block-diagram illustration for the integration of microelectromechanical system (MEMS) and metamaterials: MEMS actuated metamaterials and plasmonically enhanced physical sensors. Milestones for the former include tunable emitter/absorber [40], hologram [41], tunable waveplate [41], and metalens [42] while milestones for the latter include IR detector [43], spectrometer [44], strain sensor [45] and photoswitch [46]. 
demonstrating advanced tunability in infrared emitters/ absorbers [39], holograms [40], waveplates [41], and metalens [42]. The plasmonically enhanced physical sensor makes use of the engineered spectral response from metamaterials, such as extraordinary optical transmission, wavelength selectivity, and enhanced absorption. Milestones include metamaterial integrated IR detector [43], spectrometer [44], strain sensor [45] and photoswitch [46]. Furthermore, we note that there could be certain ambiguities when the terms "metamaterials" and "plasmon" are used. Strictly speaking, metamaterials are defined as artificial materials with engineered effective permeability and permittivity, while plasmon is a phenomenon of the collective oscillation of electrons and photons. However, since the unit cells of many metamaterials are plasmonic structures, plasmonics are sometimes considered as one type of metamaterials. Conventionally, either term can be adopted based on the specific applications. For example, the term "metamaterials" may be preferred when far-field spectra are concerned, while "plasmon" can better illustrate the near field localization for enhanced absorption or sensing applications.

\subsection{Theories and models of metamaterials}

The key to designing tunable meta-devices with MEMS technology is a comprehensive understanding of the metamaterials. So far, the available theoretical methods include lumped element circuit model [47-50], temporal coupled-mode theory [51, 52], interference theory [53, 54], etc. With their advantages and disadvantages, these methods may be preferred for different aims. For example, the lumped element circuit model allows $a b$ initio calculation of the resonance frequency, bandwidth, and extinction from the materials and structure geometries, but the calculation process could be tedious and inaccurate due to the non-uniform field charge and current distribution in metamaterials. On the other hand, temporal coupled-mode theory further simplifies the problem into a model that only involves a few general parameters such as coupling strength, external and internal damping rates, making it useful for coupled systems with multiple resonators [55-57]. Nevertheless, these parameters usually cannot be calculated directly but need to be extracted from simulation or experiments. In addition to the above two methods, metamaterials with planar structure may also be explained by the traditional interference theory in optics if the near-field coupling is negligible in the system. Finally, despite the insights and valuable design rules offered by these theoretical methods, the accurate calculation is still heavily dependent on numerical tools based on the finite-difference time-domain method, finite element method, etc. In the following, we will present a more detailed discussion about these theoretical and numerical methods.

\subsubsection{Lumped equivalent circuit model}

The lumped element model has been widely used in electronics, mechanical multibody systems, heat transfer, acoustics, etc. because it can provide a simplified understanding of these spatially distributed systems. Especially, microelectronic devices can usually be modeled into a lumped $R L C$ circuit that consists of three fundamental elements, i. e., capacitor, inductor, and resistor. In metamaterials, charges will be driven and displaced by the electric field component of the incident light, forming a dipole, which can be regarded as a micro/nano-scale capacitor. Note that the external field is oscillating, currents flow inside metamaterials, where the inductance should be considered. Lastly, the incident energy could be dissipated through either Ohmic loss in metals or dielectric absorption in the surrounding medium, which plays the role of the resistor in the electronic circuit. Therefore, metamaterials can be naturally modeled with an $R L C$ circuit [48]. Considering a simple case where these elements are connected in series. The resonance frequency, bandwidth, and quality $(Q)$-factor are:

$$
\begin{gathered}
f=\frac{1}{2 \pi \sqrt{L C}} \\
\Delta f=\frac{1}{2 \pi} \cdot \frac{R}{L} \\
Q=\frac{1}{R} \cdot \sqrt{\frac{L}{C}}
\end{gathered}
$$

Under illumination from free space, the reflection can be calculated through the conventional transmission-line model:

$$
R=\left|\frac{Z-Z_{0}}{Z+Z_{0}}\right|^{2}
$$

where $Z_{0}=377 \Omega$ is the impedance of free space. $Z$ is the impedance of the equivalent circuit $Z=R+j \cdot 2 \pi f L+1 /(j \cdot 2 \pi f C)$. In a more general case, the elements may also be connected in a more complicated way. An effective impedance should then be considered. As an example, Chen et. al. reported a tunable terahertz metamaterial using split-ring resonators (SRR) as shown in Figure 2A [58]. The equivalent circuit is sketched in 

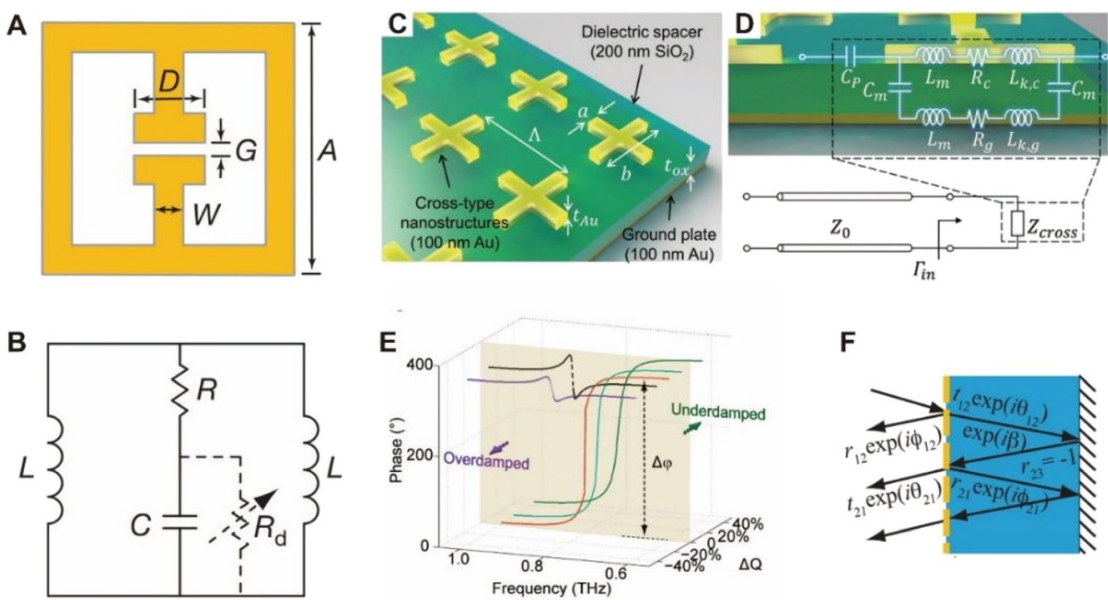

Figure 2: (A, B) Interpretation of a tunable metamaterial with a lumped element circuit model. $D, G, W$ and $A$ are the dimensions of designed split-ring resonator (SRR). $L, C$, and $R$ are the lumped elements, i. e., inductor, capacitor and resistance, respectively. $R_{d}$ is the additional tunable resistance which can be controlled by external bias [58]. (C, D) Modeling of a metamaterial perfect absorber with lumped element circuit. The circuit is constructed with only the information on materials and structure dimensions [50]. (E) Analysis of plasmonic resonance in the viewpoint of temporal coupled-mode theory. By tuning the absorptive and radiative losses, a phase transition can be obtained between

the overdamped and underdamped regions [59]. (F) Interference theory of metamaterial perfect absorber. $r_{12}, r_{21}, r_{23}, t_{12}, t_{21}$ represent the amplitude of reflection and transmission coefficients. $\Phi_{12}, \Phi_{21}, \theta_{12}, \theta_{21}$ denote the phase of reflection and transmission coefficients. $\beta$ is the propagation phase [54].

Figure 2B. In their design, an additional switchable resistor can be introduced into the circuit by controlling the depletion layer of the substrate via external bias. The existence of this additional resistor certainly affects the effective impedance of the equivalent circuit, leading to a large modulation of $\mathrm{THz}$ transmission by $50 \%$. This lumped equivalent circuit model is widely applied in the analysis of MEMS actuated metamaterials, especially the pre-stressed cantilever-based metamaterials. The unit cell of this type of metamaterial is a bimorph cantilever, where the free end bends up and the other end is anchored at the substrate. As in Eq. (1), the resonance frequency is decided by the effective inductance and capacitance. The former mainly comes from the metallic cantilever while the latter is dominated by the air gap between cantilever and substrate. When the cantilever is bent down by external stimuli, the effective capacitance and inductance are varied due to the change in the air gap and projected length of cantilever, forming variable parts of the LC circuit.

The disadvantage here is that the calculation of the values of these elements is usually not straightforward. Note that the charges and currents are oscillating in several terahertz to hundreds of terahertz frequency, several effects including non-uniform charge/current distribution and penetration in metal should be seriously considered. Consequently, accurate closed-form formulas are usually not available for the calculation of $R, L$, and $C$. Instead, effective dimensions should be estimated, for example, by adding an empirical scaling factor. Besides, the mutual coupling between the neighboring elements should also be considered to have good matching with simulated or measured results. Figure 2C, D show an example of metamaterial perfect absorber which can be accurately modeled with an equivalent circuit model by considering an effective length and mutual coupling between neighboring elements [50].

\subsubsection{Temporal coupled-mode theory}

Another important method that has been widely used in metamaterials is the temporal coupled-mode theory. Unlike the lumped equivalent circuit model, the temporal coupled-mode theory further simplifies the systems into multiple resonant modes with mutual interactions. Since this method deals with mode instead of elements, it provides clear and intuitive physical insights that are especially useful in exploring new phenomena. Starting from the simplest case where only one resonator with one port exists, we can write the fundamental equations as [55]:

$$
\begin{gathered}
\frac{d a}{d t}=j \omega_{0} a-\left(\frac{1}{\tau_{0}}+\frac{1}{\tau_{e}}\right) a+\sqrt{\frac{2}{\tau_{e}}} \cdot s_{+} \\
s_{-}=s_{+}+\sqrt{\frac{2}{\tau_{e}}} \cdot a
\end{gathered}
$$

where $|a|^{2}$ is the energy stored in the resonator, $\left|s_{+}\right|^{2}$ and $\left|s_{-}\right|^{2}$ are the power of incident light and reflected light, respectively. $1 / \tau_{0}$ and $1 / \tau_{e}$ are the internal and external decay rates of the mode. $\omega_{0}$ is the resonance frequency of the mode. At a certain frequency $\omega$, the reflection coefficient can be solved as 


$$
\Gamma=\frac{s_{-}}{S_{+}}=\frac{1 / \tau_{e}-1 / \tau_{0}-j\left(\omega-\omega_{0}\right)}{1 / \tau_{e}+1 / \tau_{0}-j\left(\omega-\omega_{0}\right)}
$$

From the equation, we can see that the perfect absorber at the resonance frequency $\left(\omega=\omega_{0}\right)$ is achieved at a critical coupling condition $\left(1 / \tau_{0}=1 / \tau_{e}\right)$. Besides, we can obtain the modulated phase $\varphi=\arg (\Gamma)$. This theory provides another perspective to understand the pre-stressed cantileverbased metamaterials, besides the above-mentioned lumped equivalent circuit model. To form a one port singlemode resonator, the metal-insulator-metal (MIM) cavity is employed while the metamaterial on the top metal layer is the bimorph cantilever. This electrostatically actuated cantilever manipulates the radiative loss dynamically and the intrinsic absorptive loss is fixed. By tuning the relationship between the two losses, an active phase transition can be demonstrated as shown in Figure 2E [59]. Interestingly, in the underdamped region $\left(Q_{a}=\omega_{0} \tau_{0} / 2>Q_{r}=\omega_{0} \tau_{e}\right)$ 2 ), the phase of $\Gamma$ changes by $2 \pi$ for sweeping $\omega$; but in the overdamped region $\left(Q_{a}=\omega_{0} \tau_{0} / 2<Q_{r}=\omega_{0} \tau_{e} / 2\right)$, the phase of $\Gamma$ changes less than $\pi$.

A drawback of the coupled-mode theory is that the relevant parameters including resonance frequency, internal and external damping rates, need to be extracted from numerical simulation or calculated by the lumped element model. Even so, this method provides valuable physical insights that can guide the design process which is especially useful in coupled systems. For example, the coupled-mode theory has been successfully used in explaining electromagnetically induced transparency (EIT) $[52,60]$, Fano resonance $[56,61]$ and plasmonically enhanced molecule sensors [57].

\subsubsection{Interference theory}

In most cases, metamaterials consist of multiple layers that have mutual interactions through the far-field interference and near-field evanescent coupling. In the cases of negligible near-field couplings, metamaterials can be conveniently modeled by the traditional interference theory [53, 54]. This method is especially suitable for materials with multilayer structures. As the first step of the interference theory, the complex transmission and reflection coefficients of each interface are calculated from Fresnel equations or extracted from simulations. Secondly, the total far-field response is the interference of all the light field after considering multiple internal reflections. Surprisingly, although the magnetic mode and near-field coupling have been believed to play a role in metamaterial perfect absorber, Chen demonstrated that this phenomenon can be explained by the interference theory, as shown in Figure 2F. $\tilde{r}_{12}, \tilde{r}_{12}, \tilde{t}_{12}$ and $\tilde{t}_{21}$ are the complex transmission and reflection coefficients. The complex nature of the coefficients indicates a phase discontinuity at the interfaces which is dependent on the resonance of plasmons. $\beta$ is the propagation phase. Note that the reflection coefficient of the back reflector is -1 . The amplitude coefficient of overall reflection is

$$
\begin{aligned}
r & =\tilde{r}_{12}+\tilde{t}_{12} \tilde{t}_{21} \sum_{n=1}^{\infty}(\exp (j .2 \beta))^{n}(-1)^{n}\left(\tilde{r}_{21}\right)^{n-1} \\
& =\tilde{r}_{12}-\frac{\tilde{t}_{12} \tilde{t}_{21} \exp (j .2 \beta)}{1+\tilde{r}_{21} \exp (j .2 \beta)}
\end{aligned}
$$

The involved parameters can be extracted from simulations by removing the back reflector. This work reports a good match between the interference theory and simulated results from numerical tools. The advantage of this method is to understand the response of metamaterials in a traditional framework. However, it is only valid at the absence of near-field coupling. Besides, the interference theory does not provide clear guidelines for structure design or materials selection. Lastly, these complex transmission and reflection coefficients at each interface cannot be calculated straightforwardly but have to be extracted from simulations. Therefore, although the interference theory provides an important viewpoint of metamaterials, it faces some intrinsic weaknesses for practical applications. This interference theory is mostly applied to the metamaterial absorbers of multiple layers, which play a crucial part in the MEMS infrared radiation sensor by enabling wavelength selectivity and enhancing detectivity.

\subsubsection{Numerical modeling}

Although the above theoretical frameworks have achieved great success in providing valuable physical insights and guidelines for design, accurate modeling of metamaterials is still heavily dependent on the numerical tools [51]. Currently, there have been many commercial software in the market for this purpose. This software provides user-friendly interfaces and integrated packages. However, special care should also be paid to obtain the correct results. For example, metals are usually regarded as perfect electric conductor (PEC) in $\mathrm{THz}$ range, but this could be wrong in shorter wavelengths such as infrared or visible range. In such regions, Ohmic loss of metals becomes not negligible, and complex permittivity should be considered in the simulation. Besides, the proper mesh is also important to avoid either too heavy computation load or artifacts due to rough mesh. 
The numerical tools provide both the far-field and near-field information. While far-field spectra are more important for practical applications, near-field plays a critical role in identifying the resonance mode and investigating the mutual coupling. The near-field information includes the distribution of electric field, magnetic field, charge, and current [62-64].

\subsection{MEMS actuation methods for metamaterials}

Because the geometric structures of the unit cell decide the effective properties of metamaterials, the most efficient tuning method is to reconfigure these unit cells, which is realized by the MEMS actuators. The in-plane and out-of-plane displacement, as well as the rotation, can be introduced by four actuator schemes: electrostatic actuation, electrothermal actuation, piezoelectric actuation, and electromagnetic actuation. Each of them has its intrinsic advantages and disadvantages. The electrostatic actuator features fast response, simplicity in fabrication and large travel distance. Its basic structure can be simplified as two closely-placed electrodes with one stationary and the other moveable. When the voltage is applied, the Coulomb force actuates the moveable electrodes towards the stationary one and is balanced by the restoring forces from the supporting spring. Therefore, the final displacement is determined. Another important feature of the electrostatic actuator is the pull-in or socalled "snap-down" effect, which refers to a sudden physical contact between two electrodes when the moveable electrode travels $1 / 3$ of the capacitive gap [65]. This pull-in effect can be leveraged for the on-off switch in metamaterials [39, 66]. Electrostatic actuators can be classified into parallel plates and interdigitated comb drives, which can respectively generate out-of-plane and in-plane displacements $[67,68]$. These two types of electrostatic actuators are widely adopted by various MEMS tunable metamaterials because they are easy to fabricate. The parallel plates usually consist of a fixed bottom plate and a movable top plate, between which there is an air gap. Surface micromachining and bulk micromachining are the two typical fabrication methods to create such an air gap, as illustrated in Figure 3A,B. In the surface micromachining method, silicon dioxide $\left(\mathrm{SiO}_{2}\right)$ is usually deposited as the sacrificial layer which is etched away later by the hydrofluoric acid (HF). If $\mathrm{SiO}_{2}$ etching is performed in the HF solution, a critical point dryer process is required to avoid the stiction, where the top membrane touches down due to the capillary force. Another choice is to perform the etching by HF vapor [69]. In the bulk micromachining method, the backside silicon (Si) from the handle wafer is usually fully etched in the potassium hydroxide $(\mathrm{KOH})$ solution. Then this released movable chip is bonded with another unreleased stationery chip. As the bonding interface and spacer, photoresist (typically SU8), polyimide and metal can be deposited or coated on the unreleased chip. In addition to this out-ofplane parallel plate structure, the in-plane comb drive is usually fabricated from silicon-on-insulator (SOI) wafers, as illustrated in Figure 3C. The frontside $\mathrm{Si}$ is firstly

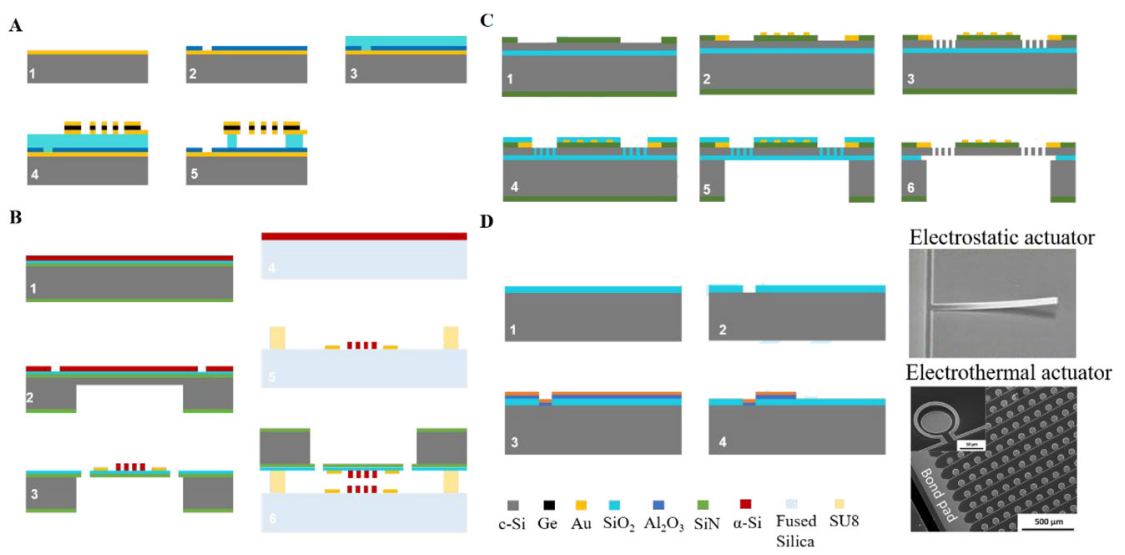

Figure 3: Fabrication process for several typical MEMS actuated metamaterials. The number indicates the sequence in fabrication. (A) Electrostatically actuated parallel plate fabricated from the surface micromachining method. It works as a reconfigurable metamaterial infrared emitter. The MIM structures are stackdeposited and the $\mathrm{SiO}_{2}$ is deposited as the sacrificial layer, which is etched away for the air gap [39]. (B) Electrostatically actuated parallel plate fabricated from bulk micromachining method. It works as the tunable dielectric metalens. The doublets are fabricated separately and then bonded together with SU8 as the bonding interface.

The backside of the movable doublet is released [42]. (C) Comb-drive actuated metamaterials. Firstly on the front side, the electrodes and metamaterials are deposited and $\mathrm{Si}$ is patterned into the comb drive. Then a protection layer of $\mathrm{SiO}_{2}$ is deposited. Finally, the backside is released for the movement [85]. (D) Pre-stressed cantilever based tunable metamaterials. The cantilever anchors are firstly defined on the sacrificial layer of $\mathrm{SiO}_{2}$, followed by the deposition of $\mathrm{Al}_{2} \mathrm{O}_{3}$ and $\mathrm{Al}$. The residue stress from the bimorph bends up the cantilever after $\mathrm{SiO}_{2}$ is released. The cantilever can be actuated electrostatically or electrothermally [70, 75]. c-Si: crystalline silicon. $\alpha$-Si: amorphous silicon. 
patterned into combs, which are then protected by deposited $\mathrm{SiO}_{2}$ in the following backside release process. Finally, the protection layer of $\mathrm{SiO}_{2}$ and the buried oxide layer are etched away together by HF.

The electrothermal actuator is another common actuating method in MEMS tunable metamaterials. It usually consists of a pair of stacked materials with different thermal expansion coefficients (TEC), such as aluminum oxide $\left(\mathrm{Al}_{2} \mathrm{O}_{3}\right)$ and $\mathrm{Si}$ [70], silicon nitride (SiN) and gold $(\mathrm{Au})$ [71]. The major features are large actuation force, slow response, and high power consumption. Although in-plane displacements can also be realized by the U-shape thermal actuators [72, 73], most of the electrothermal actuators in metamaterials are cantilevers or rings that leverage residue stress to realize out-of-plane movements [70, 74]. Specifically as shown in Figure 3D, a sacrificial layer of $\mathrm{SiO}_{2}$ is deposited beneath the bimorph and the residue stress builds up because bimorph materials are deposited at different elevated temperatures and then cooled down to room temperature. After the removal of the sacrificial layer, the mismatch in TEC manifested itself as the initial vertical displacement at the free end. The temperature variation from the current flow or the ambient environment leads to the different expansion of each material layer and thus the deformation towards the material with smaller TEC $[75,76]$. It is also feasible to bend down the suspended cantilevers or rings by electrostatic forces. A fundamental study has been performed to characterize how the geometrical parameters of cantilevers affect the residue stress and thus the tip displacements [70].

Comparing to the electrostatic and electrothermal actuators, piezoelectric actuators and electromagnetic actuators are less employed. In the piezoelectric actuator, an applied DC voltage across the electrodes of a piezoelectric material, such as lithium niobate $\left(\mathrm{LiNbO}_{3}\right)$, aluminum nitride (AlN), zinc oxide ( $\mathrm{ZnO}$ ) and lead zirconate titanate (PZT), results in a net strain that is proportional to the magnitude of the electric field and leads to the displacement. Although the piezoelectric actuator can also tune the unit cell of metamaterials [77], it causes unnecessary difficulties in the wafer-level fabrication without introducing unique advantages so that it is hardly employed as the actuating method. However, the piezoactuated MEMS metamaterials can be applied to portable and compact devices when high density integration is not necessary. The electromagnetic actuators, which involve non-COMS materials $[78,79]$, are also rare for similar reasons. Besides, microfluidics technology is also a practical method to guide the liquid metal as the unit cell [80-82]. The pneumatic valve can vary the shape of each unit cell to tailor the response of the incident EM wave [83, 84].

\section{MEMS actuated metamaterials}

\subsection{Modulation of amplitude, frequency, and polarization}

In the early stage of the MEMS actuated metamaterials, most of the demonstrations modulate EM waves homogeneously across the whole metamaterials. The unit cells at different reconfiguration states tune the amplitude [8689], frequency [75, 90-92] and bandwidth [93]. The very first MEMS reconfigurable metamaterial was demonstrated in 2009 by Tao et al [71]. The transmission is modulated by the stimulus of external temperature. As described by Figure 4A, an array of SRRs is attached to a freestanding substrate, which is supported by bimorph cantilevers of $\mathrm{Au}$ and SiN. The SRRs are in-plane at the initial state and bend upwards upon the increase of ambient temperature, because of the mismatch in the TEC of Au and SiN. When the magnetic field $(\mathrm{H})$ of incident EM waves is parallel to the gap in SRRs, magnetic dipoles are driven. The resonance strength is increased when the SRRs bend up and a larger area is pierced by the magnetic field, resulting in a decrease in the transmission. When the electric field (E) is parallel to the gap in SRRs, the upwards movement decreases the projection of the electric field and thus the circulating currents also decrease. This leads to an increase in the transmission, as shown in Figure 4B. Besides this unidirectional displacement, a bidirectional pre-stressed cantilever is modulated from 77 to $400 \mathrm{~K}$, achieving a frequency tuning of $110 \mathrm{GHz}$ and a quality factor tuning of 35\% [76]. The major application of the amplitude modulation is switch. An isotropic switch was demonstrated by stress beam based octagon rings while an anisotropic digital metamaterial was proposed to switch either only $\mathrm{x}$ polarized incidence or only y-polarized incidence or both $\mathrm{x}$ - and $\mathrm{y}$-polarized incidence or neither $\mathrm{x}$ - nor $\mathrm{y}$-polarization of incidence $\mathrm{THz}$ waves, gaining full control over anisotropy [66, 94].

Figure $4 \mathrm{C}$ is an example of the comb-drive actuated metamaterials [95]. Its unit cell is a pair of asymmetric splitring resonators (ASRRs) with one placed on a fixed post and the other on a movable frame. Three states (face-touch, separate and back-touch) are defined based on their relative positions. Under the illumination of TE waves (H parallel to the gap in ASRRs), the face-touch state shows no LC resonance because no current loop forms. The surface currents on the contact arms cancel each other and the 

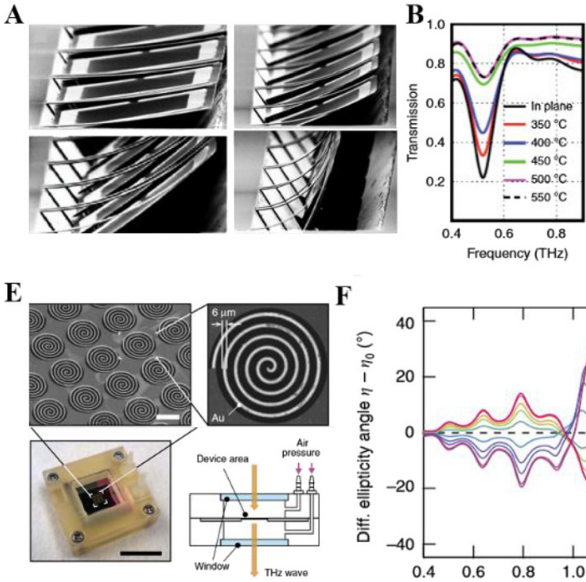

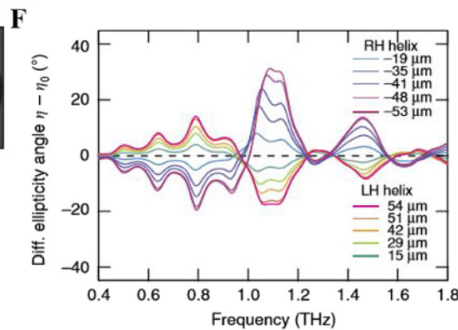

D
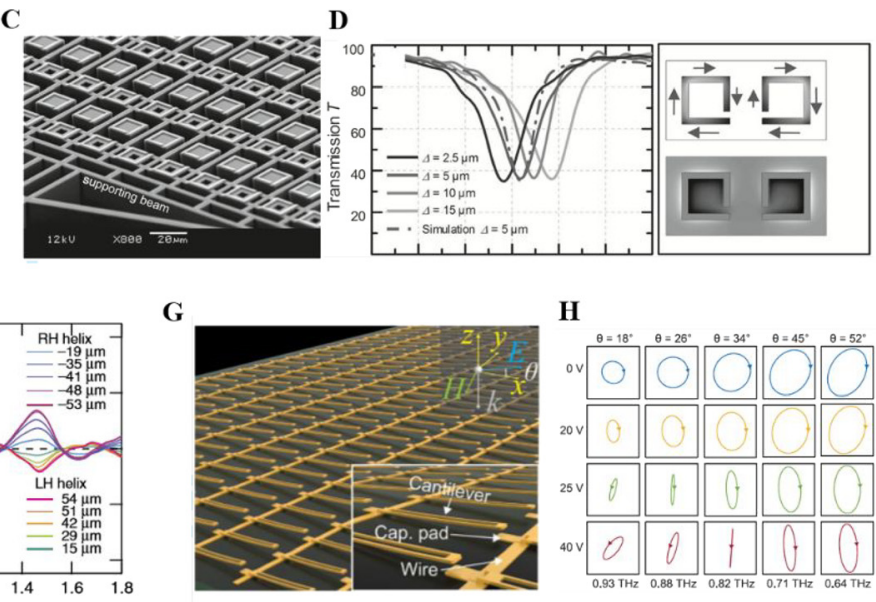

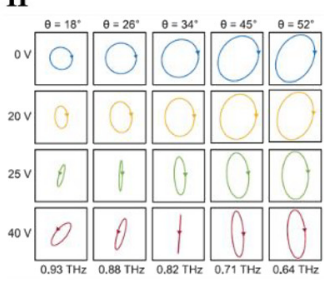

Figure 4: (A) Scanning electron microscope (SEM) image of the first thermally reconfigurable SRR metamaterial based on the pre-stressed beam and (B) its modulation in the amplitude [71]. (C) SEM image of a comb-drive actuated ASRR metamaterial and (D) its modulation in the frequency and surface current distribution at the corresponding displacement [95]. (E) Pneumatic-actuated helix metamaterials placed inside a pressure chamber and (E) its modulation in the polarization [84]. (G) Schematic of a pre-stressed cantilever based metamaterial and (H) its application in quarter waveplates [41].

currents on the outer arms oscillate parallelly. In the separate state as in Figure 4D, magnetic dipoles are excited in both ASRRs and the surface currents oscillate in phase, resulting in a dip of quality factor of 13.8. The larger separation distance blueshifts the resonance frequency. At the back-touch state, the currents on the contact arms cancel each other but the currents on the outer arms form a loop, leading to a broad dip. Under the illumination of TM waves (E parallel to the gap in ASRRs), the surface currents are always excited by the electric field while the net of magnetic dipoles is zero. The resonance peak with a quality factor of 7.8 redshifts with the increasing distance. A frequency tuning of $31 \%$ for TE polarization and $22 \%$ for TM polarization is achieved. In general, the comb-drive actuated metamaterials reconfigure the structures within or between meta-atoms by introducing lateral displacements [96-101]. Such a displacement either breaks the symmetry and leads to polarization-dependent behaviors, or changes the surface currents and leads to the modulations of amplitude and frequency. Zhao et al vertically stacked two layers of SRRs with an air gap and the top suspended layer is actuated by the comb drive [85]. Altering the coupling distance between two SRRs layers varies the mutual conductance and mutual inductance, leading to a $74 \%$ modulation in the transmission amplitude and $60(50) \mathrm{GHz}$ blueshift (redshift) in symmetric (asymmetric) mode.

More advanced demonstrations involve the polarization conversion of EM waves. The asymmetrically structured unit cells introduce anisotropic behaviors for different polarization angles and imposes different responses in the phase and amplitude. A pneumatic- actuated Archimedean spiral is described in Figure 4E to rotate the polarization [84]. This helix is in-plane initially under the non-pressure condition and is bent upwards (downwards) to be left-handed (right-handed) by the high $\mathrm{N}_{2}$ pressure in the bottom (top) chamber. The advantage of this pneumatic actuation mechanism is that it does not require metal lines to provide stimuli, which may cause interferences. Meanwhile, the actuation is bidirectional with continuous displacements. When the helix is deformed upwards as left-handed, the surface currents for the left circularly polarized (LCP) light are 4.5-fold higher than those for the right circularly polarized (RCP) light. The LCP light is reflected so that the transmission difference produces ellipticity value. In Figure 4F, a maximum ellipticity angle of $28^{\circ}$ is demonstrated at 1.0 THz. In another comb-drive actuated Maltese-cross metamaterials, a fourfold symmetry is broken by moving one of the trapezoid metal beams. When this movement is aligned with the E field of incident waves, the change in capacitance coupling between the fixed and moveable beams induces $30^{\circ}$ phase differences between o-polarization and e-polarization [97].

Another application of the polarization modulation is a transmission quarter waveplate using cantilever based metamaterials, as shown in Figure 4G [41]. These cantilevers are initially bent upwards because of the residual stress and then gradually pulled down by the electrostatic force. For the x-polarization (E parallel to the cantilever), the LC resonance is excited. The strong electric confinement in the gap between cantilever tips and substrates indicates that the major contribution to the overall 
capacitance is from tip-pad structures. The capacitance from the beam-pad plays a minor role. At each applied voltage, the gap between cantilever and substrate is continuously decreased, which induces the increase in capacitance and redshifts of the resonance. When the incident wave is y-polarized (E perpendicular to the cantilever), the cantilevers do not modulate transmission amplitude and phase because there is no electrical resonance. This anisotropic behavior produces the phase retardation and converts the circularly polarized light into a linear polarized light. A normally incident wave at $0.82 \mathrm{THz}$ of $34^{\circ}$ polarization angle is converted to linear polarization at the $40 \mathrm{~V}$ bias, at which the pull-in effect occurs, as shown in Figure 4H.

In addition to the modulation functions above, some other fundamental phenomena are also studied. EIT is actively controlled at $0.65 \mathrm{THz}$ by selecting the dark mode excitation pathways in a cantilever based metamaterial [102]. Two bright cut wire resonators (CWRs) are placed on both sides of the dark SRRs. EIT is activated when only one CWR is at a pull-in state and inductively coupled to the SRR. In another cantilever based metamaterial, the nearfield coupling is also actively switched by two conductively coupled SRRs [103].

\subsection{Manipulation of wavefront: metalens with tunable focus}

The metalens recently shows great potentials to replace the conventional bulky and expensive optical lens [11, 104, 105]. Instead of accumulating the phase difference by propagating through a parabolic lens, the wavefront is manipulated by the periodic subwavelength unit cells on an ultrathin substrate. The working principles of metalens are governed by the generalized laws of refraction [106],

$$
\sin \left(\theta_{t}\right) n_{t}-\sin \left(\theta_{i}\right) n_{i}=\frac{\lambda_{0}}{2 \pi} \frac{d \phi}{d x}
$$

where $\theta_{t}$ is the refraction angle; $\theta_{i}$ is the incident angle; $n_{t}$ and $n_{i}$ are the refractive index of the two media; $\lambda_{0}$ is the vacuum wavelength; $d \phi / d x$ is the gradient of the phase discontinuity along the interface. To focus the incident beam at a distance of $f$, the phase discontinuity across the metasurface needs to follow the equation below,

$$
\phi(x, y)=-\frac{2 \pi}{\lambda_{0}}\left(\sqrt{x^{2}+y^{2}+f^{2}}-f\right)
$$

If the phase discontinuity is tuned by changing the shape, orientation or position of the unit cell, a tunable metalens is realized. A flat lens with random access reconfigurable metamaterial (RARM) is demonstrated in Figure 5A [81]. Each unit cell consists of two metal gratings and an SRR, which is filled by the liquid metal mercury through microfluidic channels. Two metal gratings enhance the cross-polarization transmission due to the Fabry-Pérot resonance. A small part of the mercury is replaced by the air bubble. By changing the air and mercury pressure with pneumatic valves, the opening of the air bubbles can be varied row by row from $0^{\circ}$ to $150^{\circ}$ and $2 \pi$ phase difference along the $x$-direction is created [107]. Meanwhile, over $70 \%$ of transmission can be maintained. As a proof of concept, the focal length is adjustable from $5.1 \lambda$ to
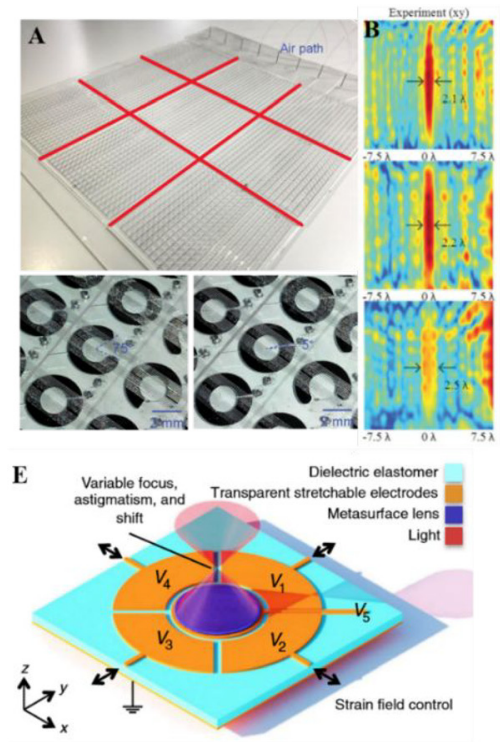
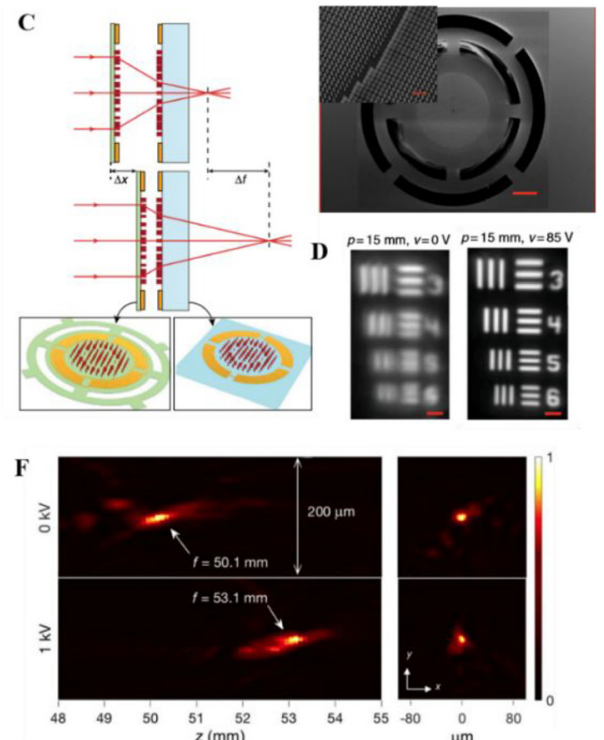

$$
\text { F }
$$

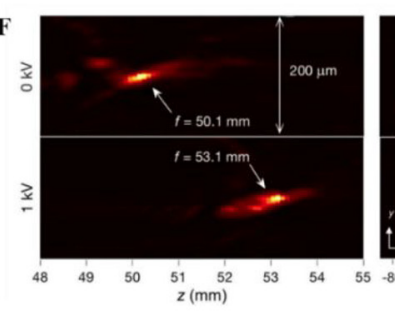

Figure 5: Metalens with tunable focal length. (A) Photography image of a random access reconfigurable metamaterial (RARM) consisting of a $60 \times 60$ square array of SRRs, partially filled with mercury. (B) Measured electric field distributions of the beam cross-section at the focal length of 5 , 10 and $15 \lambda$ (top to bottom) [81]. (C) Schematic of the MEMS-tunable dielectric metalens and SEM image of the bonded doublet. The inset is the zoom-in of the metasurface and shows the Si posts. (D) Imaging results that show the tuning of focal length [42]. (E) Schematic of the dielectric elastomer actuated (DEA) metalens with five electrodes (F) Measured intensity profiles along the propagation direction (left) and their beam crosssection at corresponding focal lengths (right) [117]. 
$15.2 \lambda$ with the full width at half maximum (FWHM) of 2.1 $\lambda$. Figure $5 B$ shows the experimental cross-section of the electric field at different focal lengths. The diffraction efficiency was measured to be around $10 \%$. A similar concept is employed in another adaptable metasurface, where the liquid metal fills the SRR and the air gap manipulates the wavefront [80]. A fixed anomalous reflection of $-45^{\circ}$ is demonstrated for normal incident waves at 10.5, 12 and $14 \mathrm{GHz}$. Besides regulating the liquid metal, microfluidics technology also can be incorporated for the liquid flow sensing [108]. The cantilever-based T-shape metamaterials are curved-up initially and then pushed down by the liquid force, which is proportional to the volumetric flow rate. The flow rate changing from 0 to $5 \mathrm{~mL} / \mathrm{min}$ corresponds to a resonance frequency shift of $0.3 \mathrm{THz}$.

In addition to the metallic metalens, all-dielectric metasurfaces attract lots of attention since dielectric materials avoid the ohmic loss and thus promise a high transmission efficiency and subwavelength resolution. A doublet of tunable focus is shown in Figure 5C [42]. The Si posts of high refractive index are deposited on both the stationary and the movable metasurfaces, which are bonded together. Two metasurfaces are designed such that a small actuation displacement between them induces a considerable change in the focal length. When $85 \mathrm{~V}$ is applied, the top metasurface moves $4 \mu \mathrm{m}$ downwards and the effective focal length is tuned from 44 to $122 \mathrm{~mm}$ as described in Figure 5D. An electrically tunable microscope consisting of three metasurfaces is also demonstrated as a prototype with ultra compactness $(1 \mathrm{~mm})$ and large corrected field of view $(500 \mu \mathrm{m})$. The scanning frequency can potentially reach a few $\mathrm{kHz}$.

Besides tuning the orientation of the unit cells and moving the doublets longitudinally, varying the relative position of each scattering element in metasurfaces also alters the phase discontinuity. One method is to fabricate the metasurface on flexible substrates and to stretch it mechanically $[109,110]$. The centrosymmetric Au nanorods are stripped into polydimethylsiloxane (PDMS) to generate a spherical wavefront [110]. By isotopically stretching the substrate and increasing the lattice distance by $30 \%$, the focal length is tuned from 150 to $250 \mu \mathrm{m}$ and the beam size is also increased from 1.6 to $2.3 \mu \mathrm{m}$ due to the decreased numerical aperture (NA). The all-dielectric counterpart is demonstrated in 2016, where the Si posts encapsulated in the elastic PDMS achieve a focal distance tuning from 600 to $1400 \mu \mathrm{m}$ [111]. More metamaterials and metasurfaces on flexible substrates are demonstrated to realize the functions of half or quarter waveplate [112] and frequency tuning [113-115].

The dielectric elastomer actuator (DEA) or "artificial muscle", which can be stretched by electric fields, is another actuation method in addition to mechanical stretchings [116]. As shown in Figure 5E, Si metasurface is bonded to the stacked layers of DEA (polyacrylate) and electrode (single-walled carbon nanotubes, SWCNT), which are both transparent in the visible range [117]. The SWCNT is transferred through masks to form five electrodes so that the DEA can be actuated in different directions. The focal length can be either increased or decreased, subjecting to the expansion or compression of the metasurface. Astigmatism and image shift can also be realized by selectively applying voltages to certain electrodes. The focal length is experimentally tuned by $15 \mathrm{~mm}(30 \%)$ under the applied voltage of $3 \mathrm{kV}$. The measured intensity profiles along the propagation direction, as well as the spot profiles at two focuses, are in Figure 5F.

\subsection{Manipulation of wavefront: beam steering}

Beam steering holds wide applications in the light detection and ranging (LiDAR), free-space optical communications and holography. The working principle of beam steering is similar to that of metalens. The phase profile is manipulated locally by each unit cell to deflect incident beams. The schematic of a $160 \times 160$ large-scale optical phased array actuated by comb drives is demonstrated in Figure 6A [118]. In each unit cell, the grating element is integrated on top of a comb drive to increase the filling factor. All these grating elements are individually addressable. Comparing to the conventional piston mirrors where $2 \pi$ phase is achieved only by the large vertical displacement of half-wavelength [119], this grating is designed to move laterally along the gratingvector direction and $2 \pi$ phase shift is realized with $955 \mathrm{~nm}$ movement. Figure 6B shows the SEM image of the optical phased array and the grating element. Holes are etched on the gratings to facilitate the wet HF release. When $10.5 \mathrm{~V}$ voltage is applied, the beams can be steered within the $6.6^{\circ} \times 4.4^{\circ}$ field of view (FOV), as shown in Figure 6C. The waterfall image demonstrates the beam profile with mainto-sidelobe suppression ratio of $10.8 \mathrm{~dB}$. The diffraction efficiency is $16 \%$ on the uncoated silicon gratings and the response time is $5.7 \mu \mathrm{s}$. Besides, by fabricating the fixedgrating arrays with predetermined lateral offsets, hologram in the far-field can also be measured. Another example of beam steering is demonstrated with the Si nanowire array, which is fabricated from the SOI platform [120]. The buried oxide is released to create a suspension gap. The width of the nanowire array is tapered from 80 to $160 \mathrm{~nm}$. As shown in Figure $6 \mathrm{D}$, Si nanowire supports the Mie resonance as the 


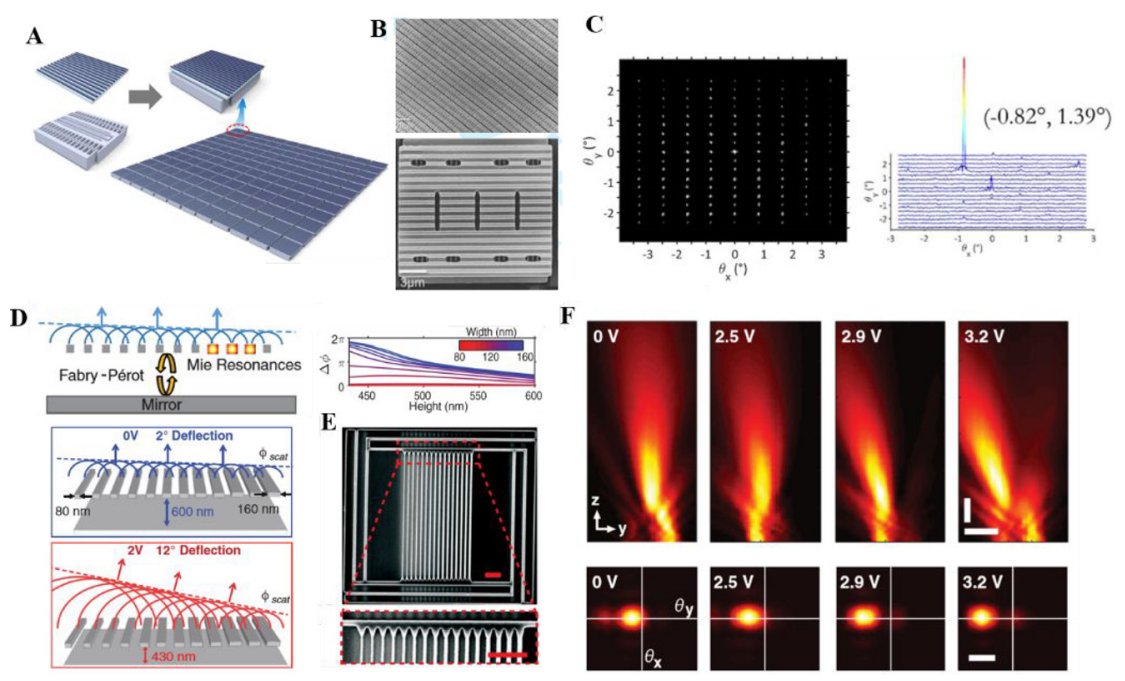

Figure 6: (A) Schematic of the MEMS grating optical phased array. The grating element is integrated on top of a comb drive in each unit cell. (B) SEM image of the optical phased array and the grating unit cell. Holes are etched on the grating for wet $\mathrm{HF}$ release. (C) Overlay of steered beams within the FOV (left) and the waterfall images showing beam profiles (right) [118]. (D) Working principle and (E) SEM image of a Si nanowire array (F) Diffracted beam profiles (top, scale bar is $3 \mu \mathrm{m}$ ) and corresponding Fourier images of the steered beams steering showing the continuous deflection from $2^{\circ}$ to $12^{\circ}$ (bottom, scale bar is $8^{\circ}$ ) [120]. individual dielectric antenna. Meanwhile, this Mie resonance is coupled with the Fabry Perot resonance that exists between the substrate and nanowire array. A $2 \pi$ reflection phase can be controlled over a broad range by tuning the gap, where the interplay between the Mie and Fabry-Pérot resonances is manipulated. The SEM image of the Si nanowire array is in Figure 6E. The actuating voltage of $3.2 \mathrm{~V}$ decreases the gap and therefore the diffracted beam is continuously redirected from $2^{\circ}$ to $12^{\circ}$. The beam profiles and their Fourier images in the angular space are shown in Figure 6F. The operation frequency is expected to be as high as $1 \mathrm{MHz}$. This device does not degrade noticeably after $10^{9}$ cycles, showing excellence robustness. This is also a versatile platform, which can also realize the temporal color mixing and light focusing by varying nanowire designs.

\subsection{Tunable metasurface-based holograms}

Metasurface holograms are emerging due to their importance in the communication and information storage. A multiplexed metasurface hologram is demonstrated on the stretchable substrate in Figure 7A [40]. Its working mechanism is similar to that of the flexible metalens mentioned above [110]. The $\mathrm{Au}$ nanorods with different orientations introduce the position-dependent phase discontinuity, which manipulates the transmitted wavefront. When the phase discontinuity is changed by the isotropic stretching (stretching ratio $s$ ), the image displaying plane $f$ is shifted farther to $s^{2} f$ and the images also become bigger. Leveraging such a concept, the phase distribution is designed with the computational techniques [121] so that three images: a pentagon, a square, and a triangle are embedded simultaneously in the unstretched substrate. Their image planes are sparsely placed to avoid the interference from other image planes. As shown in Figure 7B, the stretching moves the hologram farther, crossing the observation plane. Therefore, all three images can be observed when the substrate is stretched by the ratio of $1,1.12$ and 1.3 .

Another possibility of the metamaterial hologram is proposed with the cantilever based metamaterials in $\mathrm{THz}$
A

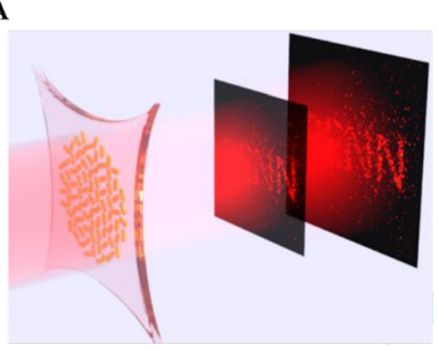

B

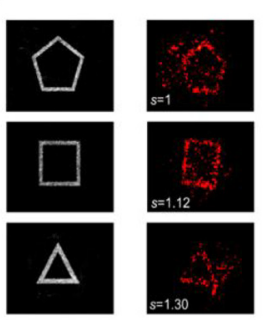

C

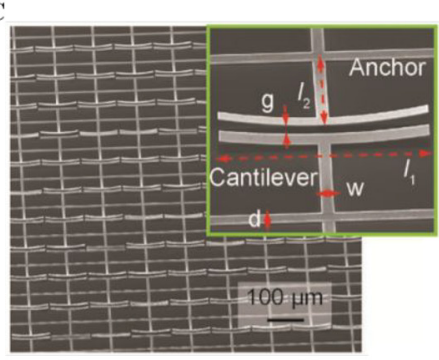

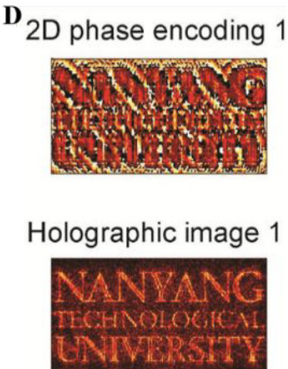

Figure 7: (A) Schematic of the multiplexed metasurface holograms on a stretchable substrate. (B) Reconstructed images that are captured at the observation plane when the substrate is stretched to different ratios [40]. (C) SEM image of the pre-stressed cantilever based metamaterial and the (D) computational results of holographic image [122]. 
as shown in Figure 7C [122]. By applying electrostatic forces, the cantilevers bend downward and each cantilever with different tip displacements can induce the phase differences from $-75^{\circ}$ to $-340^{\circ}$. With the assumption that each of the cantilever is controlled independently, this pixellevel manipulation enables real-time rewritable hologram. Figure 7D shows the holographic image of a six-digit phase encoding, which is numerically calculated by RayleighSommerfield diffraction theory.

\subsection{Tunable emitter and perfect absorber}

The tunable absorber in infrared and $\mathrm{THz}$ range is of great importance due to its applications in single-pixel imaging and spectroscopy. In the perfect absorber consisting of the MIM structures [5], changing the distance between the metamaterial elements and the ground metal substrate is a feasible method to modify its impedance and thus its resonance response. In the very first demonstration of the tunable absorber, a top layer that combines babinet metamaterials and the dielectric layer is freestanding on the metal ground with the support from eight springs [123]. The perfect absorption at $6.2 \mu \mathrm{m}$ is switched on when the top layer is brought into the physical contact with the ground at $16 \mathrm{~V}$, which is called "snap-down" state. The restoring force from the supporting springs pulls the top layer back to the free-standing state when the applied voltage is off so that the perfect absorption is switched off. The modulation index is $56 \%$. The similar MEMS tuning mechanism is applied to another $\mathrm{THz}$ perfect absorber, as shown in Figure 8A [124]. Nine mutually-coupled electric split-ring resonators (ESRRs) on a SiN membrane are supported by actuation arms. ESRRs are selected because their modes are localized, leading to a lower radiative loss and a higher quality factor. The resonance shift of $165 \mathrm{GHz}$ and an absolute absorption change of $65 \%$ are achieved at a snapdown state under the voltage of $250 \mathrm{~V}$, as illustrated in Figure 8B. However, in this case the snap-down irreversibly damages the devices because of the lack of anti-stiction layers.

On the contrary, the perfect absorber can also work as an emitter according to Kirchhoff's law. The ability to control the emissivity of materials has been highly desired because of its various applications in energy harvesting and thermal control. In the conventional thermal emitters, the emissivity is temperature independent and the radiation is tuned by the device temperature based on Stefan-Boltzmann law. Therefore, the disadvantages include high-temperature operation, slow modulation speed and lack of wavelengthdependent emissivity. The metamaterial has the potential to overcome this bottleneck since the artificially engineered geometry instead of composites determines the emissivity [125]. The incorporation of MEMS actuators offers the highspeed modulation of the thermal emission (around tens of $\mathrm{kHz}$ ). Leveraging the same structures as the perfect absorber, a reconfigurable metamaterial emitter is realized in Figure $8 \mathrm{C}$ [39]. The absorptivity at $8.9 \mu \mathrm{m}$ is tuned by $70 \%$ in Figure 8D and the inset shows the optical image of the devices at free-standing and snap-down states. The emitted power density is measured as $12.9 \mathrm{Wm}^{-2}$ at off-state (freestanding) and $16.9 \mathrm{Wm}^{-2}$ at on-state (snap-down). If comparing to the conventional thermal emitters, this tuning in emitted power density can be achieved equivalently by changing the temperature of $18.9^{\circ} \mathrm{C}$. Then this emitter is made into an $8 \times 8$ array to display the letter " $D$ " as a proof of
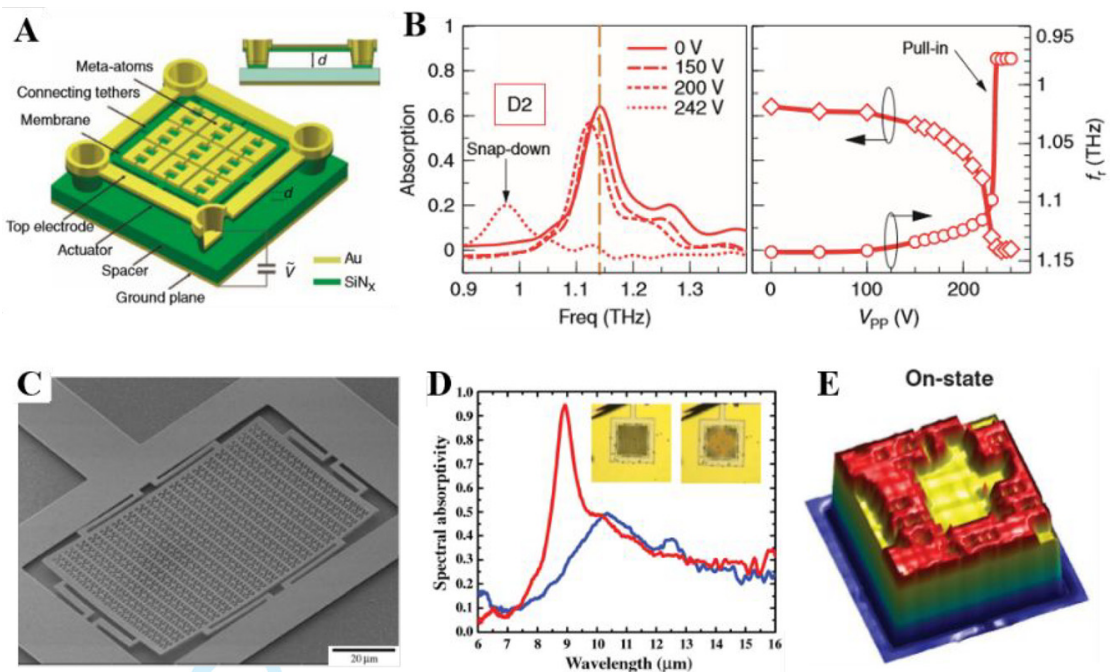

Figure 8: (A). Schematic of the THz tunable electric split-ring resonators (ESRRs). The distance $d$ between the metamaterial layer and the substrate can be tuned electrostatically. (B) Measured absorption at different driving voltages and snap-down states [124]. (C) SEM image of the babinet metamaterial-based tunable infrared emitter (D) Measured absorptivity at the freestanding state and snap-down state. Optical microscope (OM) images of these two states are in the inset. (E) An $8 \times 8$ array of the tunable emitter displaying letter " $D$ " for the battlefield identification [39]. 
concept for battlefield identification (Figure 8E). Another thermochromic infrared metamaterial is also demonstrated using babinet structures [126]. Instead of being actuated by the electrostatic force, these metamaterial elements are supported by the bimorph arms of $\mathrm{Au}$ and SiN. The increased ambient temperature bends down the metamaterial layer due to the difference in the TEC of Au and SiN, thus the spectral emissivity is monolithically increased. The peak emissivity is tuned from 0.5 (at $4.8 \mu \mathrm{m}$, room temperature) to 0.81 (at $5.2 \mu \mathrm{m}, 623 \mathrm{~K}$ ). From 273 to $623 \mathrm{~K}$, its power density is increased by 215 times while the counterpart from blackbody radiation is increased by only 90 times.

The major challenge for this out-of-plane actuation scheme is to maintain the metamaterial layer flat during movements. The imperfections in the fabrication, such as the non-uniform deposition of the sacrificial layer and the residue stress caused by the deposition recipe, may lead to the tilting and curvature of the freestanding layer [124]. The design of actuators has a significant influence on the flatness of the top membranes and the actuating voltage. The curvature of the metamaterial layer may induce the nonuniform emissivity or absorptivity across the membrane and degrade the overall performances.

\subsection{Logic operation}

The logic operations in $\mathrm{THz}$ are realized by stress-beam based metamaterials [127, 128]. In Figure 9A, its unit cell comprises of two SRRs laterally separated by a gap, which are bent up initially and can be controlled independently as two electric inputs [127]. The sharp and weak Fano resonance at $0.56 \mathrm{THz}$ is excited and tuned by the asymmetric structure when one SRR is snapped down into the contact with the substrate and the other one is gradually pulled down by the electrostatic force. The exclusive-OR (XOR) is demonstrated in Figure 9B. The pull-in voltage (35 V) and zero applied voltage represent binary inputs " 1 " and " 0 " respectively, while the Fano resonance amplitude in the farfield represents the optical binary outputs " 1 " and "0".
Exclusive-NOR (XNOR) is exhibited by the Q-factors of the Fano resonance. Besides the characterization of far-field amplitude, the near-field electric amplitude between two cantilever tips also reveals the NAND operation in the simulation. Similar concepts of binary coding are also applied in the ESRRs, which function as meta-bits for the logic gate [128]. Three input states are shown in Figure 9C while the transmission outputs analogous to NOR and AND are demonstrated at 0.26 and $0.36 \mathrm{THz}$ respectively in Figure 9D.

\section{Plasmonically enhanced physical sensor}

Besides the role of actuator which transforms electric power into mechanical motion, the MEMS technology also plays a role of sensor that transforms stimulus signals from the ambient into the electrical output. One of the MEMS physical sensors that integrate with metamaterials is the infrared or $\mathrm{THz}$ radiation detector. Unlike the III-V or 2D material based photodetector, there are no electron-hole pairs generated in the MEMS-based radiation detector. Instead, the heat generated by the incident EM wave changes the properties of the materials, which are leveraged to characterize the radiation power. The advantage is that cryogenic cooling is not required to reduce thermal noise. However, the lack of the wavelength selectivity and low absorption efficiency are the intrinsic drawbacks and necessitates the incorporation of metamaterials. Conventional MEMS radiation detectors include bolometer and thermopile [38, 129, 130]. The bolometer measures the temperature-dependent resistance while the thermopile measures the voltage output that is a result of the temperature difference between the hot junction (absorber area) and cold junction (substrate).

Figure $10 \mathrm{~A}$ is a very early demonstration of microbolometer that is integrated with metamaterials [131]. $50 \mathrm{~nm}$ conductive Si sensing layer is sandwiched between
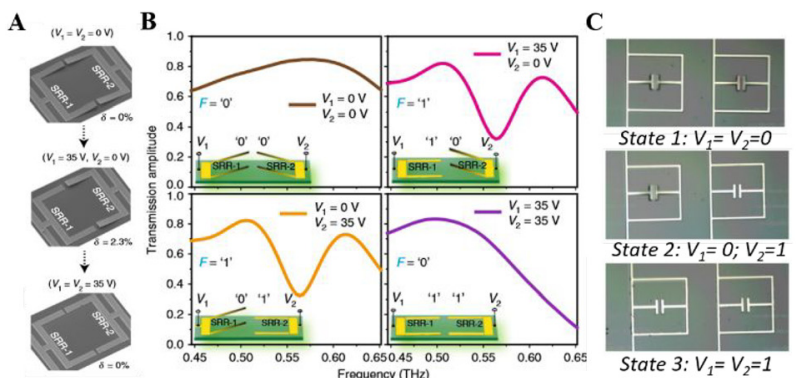

D

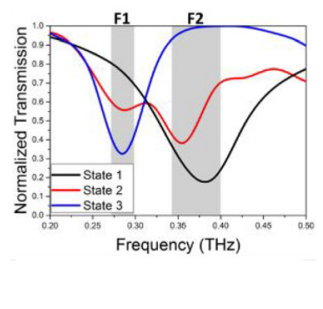

Figure 9: (A) SEM image of the unit cell where two SRRs are at different input states. (B) The Fano resonance output shows the behaviors of exclusive-OR (XOR) logic operation. The inset is the corresponding electric input [127]. (C) OM image of the ESRRs at three different input states (D) Measured transmission spectra at three states, with NOR at F1 $(0.26 \mathrm{THz})$ and AND at F2 (0.36 THz) [128]. 
two layers of SiN, on the top of which the square-shape MIM absorbers are deposited. The peak absorption of 0.8 is realized at $6.4 \mu \mathrm{m}$. The wavelength tailoring and tuning are feasible by varying the unit cell of absorbers [132]. MIM absorbers are also applicable to the thermopile [133]. Since the multilayer absorbers require precise thickness control, two-dimensional (2D) plasmonic crystals with better fabrication tolerances are employed in the thermopile in Figure 10B [134]. $250 \mathrm{~nm} \mathrm{Au}$ layer is deposited as the anisotropic nanoholes and the responsivity of $160 \mathrm{mV} / \mathrm{W}$ is achieved around $5 \mu \mathrm{m}$. The geometrical structures of this bolometer and thermopile are similar because both of them need thermal isolation to reduce the heat dissipation into the substrate. The polarization-dependent absorption can be realized by integrating the one-dimensional (1D) grating structures into the thermopile [135]. Although the lowquality factor is the intrinsic drawback of the plasmonic resonance, which limits the resolution of the infrared detector, an MIM perfect absorber is demonstrated recently with an ultranarrow band, approaching the physical limits [50]. The full-width-half-maximum (FWHM) is $3 \%$ at $5.83 \mu \mathrm{m}$ while the absorption is larger than $99.7 \%$. Based on this advancement, a prototype of a chip-scale multispectral infrared detector is realized and shows the great potential for the ultra-miniaturized spectrometer [136].

In addition to the bolometer and thermopile, the heat generated by the incident EM wave can also be converted to the displacement of bimorph cantilevers, which is read out by another off-chip probing light [141]. A focal plane array consisting of $256 \times 256$ bimorph cantilevers shows its capability for IR imaging [137]. Figure 10C is the top view and cross-section of each unit cell, which contains the MIM absorbers, bimorph cantilevers, and thermal isolation legs. To form the MIM absorbers, metals are deposited on both sides of the SiN membrane. Meanwhile, the bottom metal layer also works as a mirror to deflect the incident probing light so that the mechanical loading is reduced. The bimorph cantilevers provide out-of-plane displacements when the heat is generated from the incident EM wave and thermal isolation legs reduce heat dissipation into the substrate. During the imaging, the device is illuminated by the IR radiation and the displacement of the whole array is probed simultaneously by a beam of parallel visible light. The integration of metamaterial absorber improves absorption and imaging quality. The detection of $\mathrm{THz}$ and microwave is also demonstrated with this mechanism. An array of bimorph cantilevers integrated with SRR achieves the responsivities as high as $16,500 \mathrm{~V} / \mathrm{W}$ and noise equivalent powers of $10^{-}$ ${ }^{8} \mathrm{~W} / \mathrm{Hz}^{1 / 2}$ at single-pixel [142]. Figure 10D shows another suspended bimorph nanobeam with nanoslit nanoantennas for IR detection [138]. The noise equivalent power (NEP) is measured to be $5 \mathrm{nW} / \mathrm{Hz}^{1 / 2}$ at $1 \mathrm{kHz}$.

Besides the displacement in the bimorph cantilevers, heat from the radiation can also induce the resonance shift
A

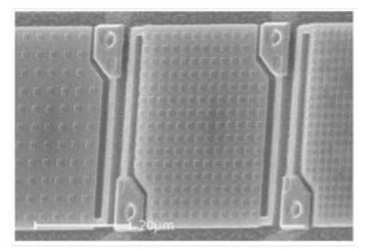

B
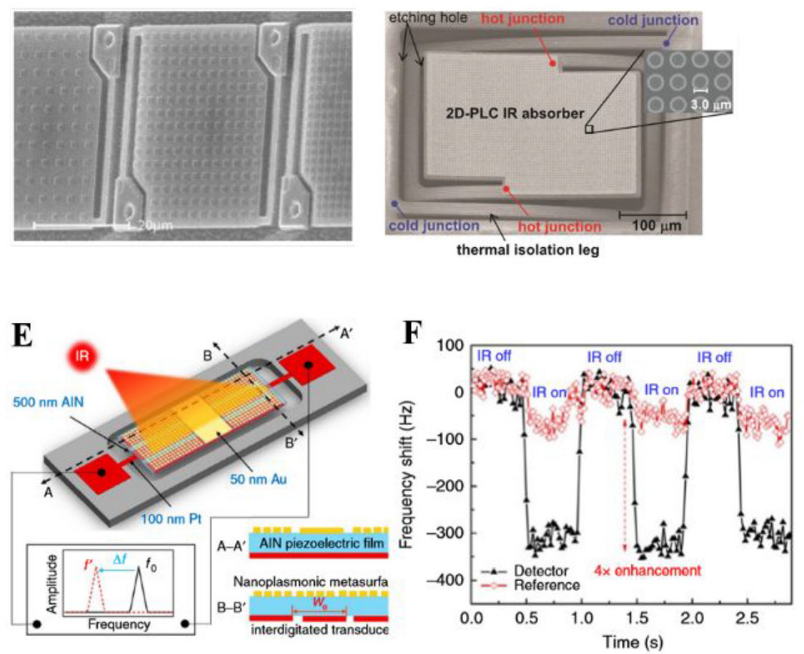

C
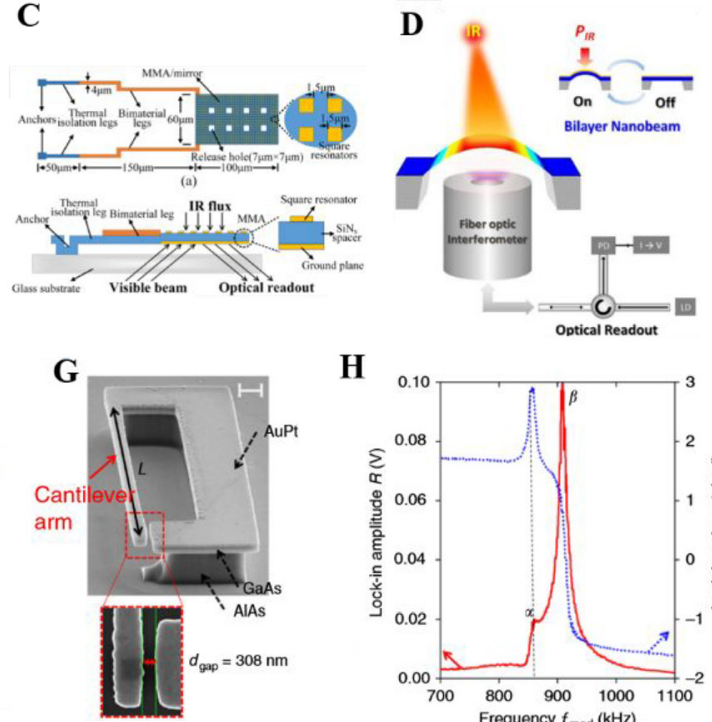

H

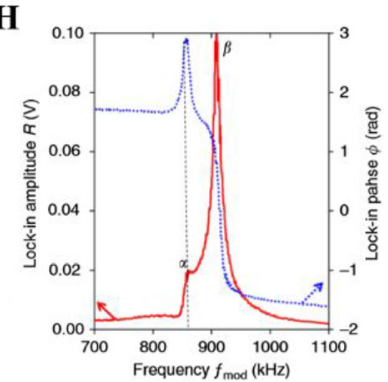

Figure 10: MEMS radiation detectors in infrared and THz range, integrated with metamaterials (A) SEM image of the microbolometers with the square MIM absorbers [131]. (B) SEM image of the thermopile with the two-dimensional plasmonic crystals [134]. (C) Schematic of the focal plane array with MIM absorbers [137]. (D). Schematic of nanobeam with nanoslit resonators [138]. (E) Schematic of the AIN piezoelectric resonator based IR detector and its $(F)$ temporal response. The comparison with dummy resonator without metamaterials shows 4-fold enhancement [139]. (G) SEM image of the meta-atom: bilayer asymmetric SRR with GaAs and metal (H) Amplitude and phase response when the THz frequency is swept around the mechanical resonances $\alpha$ and $\beta$ [140]. 
in the piezoelectric resonator [139]. In Figure 10E, the thin aluminum nitride (AlN) film is sandwiched by metals with the bottom layer as an interdigitated transducer (IDT) and top layer as square plasmonic resonators. The IDT actuates and senses the high order of vibrations (quality factor of 1116) in the AlN film while the plasmonics resonator increases the absorption to $80 \%$ around $8.8 \mu \mathrm{m}$. Comparing to the resonator without the metasurface in Figure 10F, the responsivity is enhanced by four times. The response time is $440 \mu \mathrm{s}$ and NEP is measured around $2.1 \mathrm{nW} / \mathrm{Hz}^{1 / 2}$ at the designed wavelength. Another example to detect $\mathrm{THz}$ involving optomechanics is in Figure 10G, where the single meta-atom is a bilayer asymmetric SRR of GaAs and metals [140]. By focusing the near-infrared laser onto the device, two mechanical modes are identified as the results of the Brownian motion: in-plane $(\alpha)$ and out-of-plane $(\beta)$. When the single meta-atom is illuminated by the $\mathrm{THz}$ radiation, the charges with opposite signs oscillate on both sides of the gap, creating a Coulomb force that attracts the in-plane mode. However, as in Figure $10 \mathrm{H}$, the out-of-plane mode is also varied because of the photothermal effect, where the heat deforms the bilayer cantilever. The overall signal is a linear superposition of both in-plane and out-of-plane modes.

In addition to the infrared and $\mathrm{THz}$ detector, metamaterials and plasmonics are also employed in other MEMS physical sensors. A zero-power plasmonically enhanced micromechanical photoswitch is in Figure 11A. Two symmetric cantilevers are made face to face. In each cantilever, there is a head, an inner pair of thermally sensitive bimaterial legs and an outer pair of bimaterial legs connected to the substrate. The inner and outer bilayer legs are connected by thermal isolation links. One head of the cantilever is integrated with plasmonics absorbers while the other one is covered by $\mathrm{Au}$. The head with an absorber is integrated with a bowl-shaped Pt tip. This tip is connected with one electrode and is suspended above the other contact pad, which is connected with the other electrode. When the incident radiation that matches the spectrum of plasmonic resonators impinges the absorber, heat bends down the bimorph cantilever and the Pt tip touches with the contact pad below. Therefore, the circuit is tuned from open to closed. With such a photoswitch, the sensor can remain in a dormant state with zero power consumption until being awakened by the external radiation that is related to a certain event. The threshold is as low as $500 \mathrm{nW}$ and there is no failure in 1000 consecutive switchings.

An array of rectangular gold holes that exhibits extraordinary optical transmission is leveraged as the reflector in the MEMS tunable Fabry-Pérot spectrometer in Figure 11B. These rectangular gold holes are supported by the bilayer of poly-Si and gold and are suspended on the other gold reflector. As the suspended layer is pulled down by the electrostatic force, the resonance condition shifts the dips in reflectance in Figure 11C. The decreased distance between two gold layers eliminates the higher-order resonances so that the number of dips drops. The quality
A
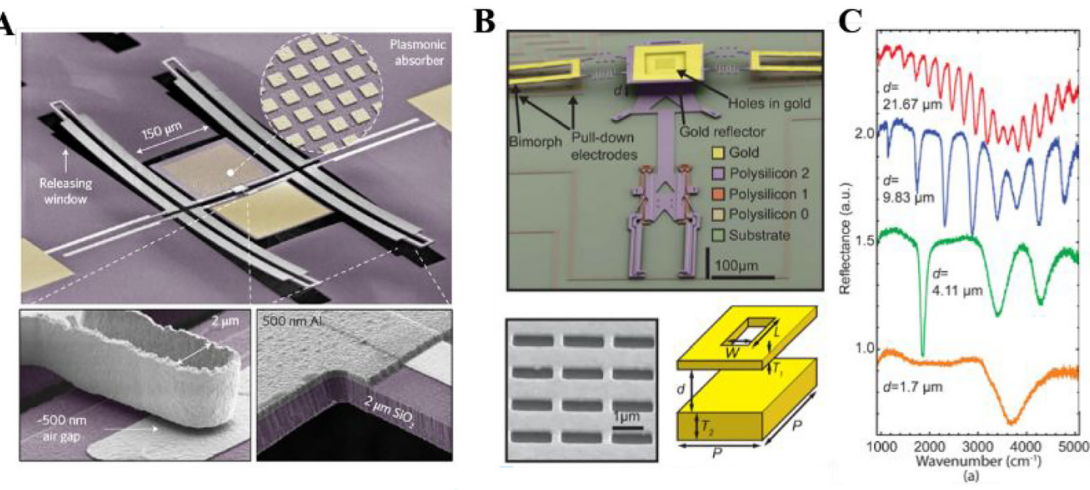

D

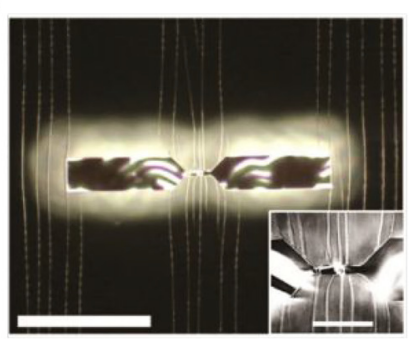

E

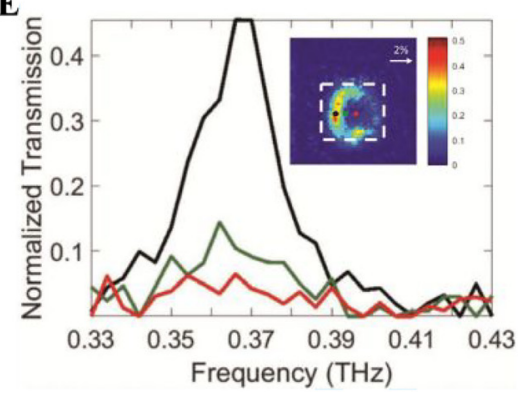

Figure 11: (A) SEM image of a zero-power plasmonically enhanced micromechanical photoswitch [46]. (B) MEMS tunable FabryPerot spectrometer that leverages extraordinary optical transmission and its (C) measured reflectance under different cavity lengths [44]. (D) Optical image of a strip dipole antenna. The inset shows a meta-atom with microcracks that are located at the edges of the two pads and the bowtie-shaped center. (E) Crosspolarimetric transmission (CPT) measured at the pixels with corresponding colors in the inset [45]. 
factor of the dip around at $3820 \mathrm{~cm}^{-1}$ is lower because the extraordinary optical transmission shows the minimum reflectance at this wavenumber.

As the counterpart of the strain-actuated metamaterials, the metamaterials on stretchable substrates can sense the external force or displacement by observing the resonance frequency shift [143-150]. An asymmetrical strip dipole antenna in Figure 11D is encapsulated in the PDMS layers to locate and record the region of stress and fatigue [45]. When the strip dipole antennas experience the strain that is higher than the designed threshold, the cross-bar junction will be irreversibly broken as shown in the inset of Figure 11D. Consequently, the cross-polarimetric transmission (CPT) is destroyed. An array of such dipole antennas is distributed across the PDMS and maps the strain history. Black, green and red lines in Figure 11E are the spectral CPT measurements at the pixels (inset) with corresponding colors. The black pixel is the position where cross-bar junctions are intact and CPT is maintained, while the green and red pixels are where junctions are broken and CPT disappears.

\section{Conclusion and outlook}

MEMS technology has been incorporated into the metamaterials and plasmonics as sensors and actuators for the past decade. In the MEMS actuated metamaterials, the electrothermal actuators and electrostatic actuators are the most widely-used methods. Thanks to the mature MEMS technology, the research in tunable metamaterials has progressed rapidly from the demonstration of fundamental modulations of frequency, amplitude, and bandwidth, to more advanced applications such as the manipulation of the wavefront, tunable emitter/perfect absorber, and logic operations. Currently, most of the demonstrated tunable metamaterials are controlled in a relatively simple way. Either there is only an on-off state for all the unit cells or the independent control of unit cells is only possible for row by row tuning $[80,122,151]$. This control method satisfies some needs like switches, waveplates and anomalous reflection that requires only $1 \mathrm{D}$ phase discontinuity. However, the rand access is highly desired. The independent control of each unit cell shows great potentials in various applications such as the dynamical beam steering, the light detection and ranging (LiDAR) and real-time rewriteable holographic imaging. Although the step from 1D control to 2D control is straightforward theoretically, engineering efforts are required to overcome the challenge in the sophisticated fabrication. Another challenge emerges when the working wavelength of metamaterial moves from the
$\mathrm{THz}$ to the infrared or even the visible range. The smaller feature size of the unit cell needs to shrink the size of actuators from the micrometer scale to the nanometer scale, which is mainly limited by the fabrication techniques.

In the plasmonically enhanced physical sensors, the most promising research direciton could be MEMS-based radiation detectors. Because of the limitation in the bandgap of popular photodetectors materials like III-V and germanium, the on-chip uncooled detector working beyond $2 \mu \mathrm{m}$ is still under research. While the $2 \mathrm{D}$ materials like graphene and black phosphorus recently emerge as solutions [152-155], the thermopiles and bolometers have been commercially available for decades. However, their performances can be further enhanced with the integration of the metamaterial perfect absorbers, which offer almost unity absorption and satisfying wavelength selectivity [50]. Comparing to the conventional nondispersive infrared system (NDIR) which involves the Fabry Perot filters [156-158], the plasmonically enhanced radiation sensors integrate the spectral filter with the detectors without significantly increasing the fabrication difficulties. It shows great potentials in the ultracompact MIR spectroscopic sensing. Besides the radiation detector, the cantilevers integrated with metamaterial absorbers can also work as the zero power photoswitch, which is turned on by the external radiation that is associated with a certain event [46]. It can find applications in the event-driven sensors, which is a necessary part of the Internet of Things (IoT).

Funding: This work was supported by NRF-CRP15-2015-02 "Piezoelectric Photonics Using CMOS Compatible AlN Technology for Enabling the Next Generation Photonics ICs and Nanosensors" (WBS: R-263000C24281) at National University of Singapore, Singapore; NRF2015-NRF-ISF0012620 "Reconfigurable data center optical interconnects using fast nanophotonic MEMS waveguide switches" (WBS: R-263000C64281) at National University of Singapore, Singapore; “THz Metamaterial Based Microemitters" (Grant No: 61474078) at National University of Singapore Suzhou Research Institute.

\section{Reference}

[1] R. A. Shelby, D. R. Smith, and S. Schultz, "Experimental verification of a negative index of refraction," Science, vol. 292, pp. 77-9, 2001. https://doi.org/10.1126/science.1058847.

[2] J. Hao, J. Wang, X. Liu, W. J. Padilla, L. Zhou, and M. Qiu, “High performance optical absorber based on a plasmonic metamaterial,” Appl. Phys. Lett., vol. 292, pp. 2511042010. https://doi.org/10.1063/1.3442904. 
[3] C. M. Watts, X. Liu, and W. J. Padilla, "Metamaterial electromagnetic wave absorbers,” Adv. Mater., vol. 292, pp. OP98-OP120, 2012. https://doi.org/10.1002/adma.201200674.

[4] N. Liu, M. Mesch, T. Weiss, M. Hentschel, and H. Giessen, "Infrared perfect absorber and its application as plasmonic sensor," Nano Lett., vol. 292, pp. 2342-8, 2010. https://doi.org/ $10.1021 / \mathrm{nl9041033.}$

[5] N. I. Landy, S. Sajuyigbe, J. J. Mock, D. R. Smith, and W. J. Padilla, “Perfect metamaterial absorber," Phys. Rev. Lett., vol. 292, pp. 207402, 2008. https://doi.org/10.1103/PhysRevLett.100. 207402.

[6] M. Choi, S. H. Lee, Y. Kim, et al., "A terahertz metamaterial with unnaturally high refractive index," Nature, vol. 292, pp. 369, 2011. https://doi.org/10.1038/nature09776.

[7] J. Gu, R. Singh, X. Liu, et al., "Active control of electromagnetically induced transparency analogue in terahertz metamaterials," Nat. Commun., vol. 292, pp. 1151, 2012. https://doi.org/10.1038/ ncomms2153.

[8] S. -Y. Chiam, R. Singh, C. Rockstuhl, F. Lederer, W. Zhang, and A. A. Bettiol, "Analogue of electromagnetically induced transparency in a terahertz metamaterial," Phys. Rev. B, vol. 292, pp. 153103, 2009. https://doi.org/10.1103/PhysRevB.80.153103.

[9] S. Wang, P. C. Wu, V. - C. Su, et al., "A broadband achromatic metalens in the visible," Nat. Nanotechnol., vol. 292, pp. 227, 2018. https://doi.org/10.1038/s41565-017-0052-4.

[10] K. Chen, Y. Feng, F. Monticone, et al., "A reconfigurable active huygens' metalens," Adv. Mater., vol. 292, pp. 1606422, 2017.

[11] X. Chen, L. Huang, H. Mühlenbernd, et al., "Dual-polarity plasmonic metalens for visible light,” Nat. Commun., vol. 292, pp. 1198, 2012. https://doi.org/10.1038/ncomms2207.

[12] Z. Wang, L. Jing, K. Yao, et al., "Origami-based reconfigurable metamaterials for tunable chirality," Adv. Mater., vol. 292, pp. 1700412, 2017. https://doi.org/10.1002/adma.201700412.

[13] W. Ma, F. Cheng, Y. Liu, "Deep-learning-enabled on-demand design of chiral metamaterials," ACS Nano, vol. 292, pp. 6326-34, 2018. https://doi.org/10.1021/acsnano.8b03569.

[14] Y. Zhao, A. N. Askarpour, L. Sun, J. Shi, X. Li, and A. Alù, "Chirality detection of enantiomers using twisted optical metamaterials," Nat. Commun., vol. 292, pp. 14180, 2017. https://doi.org/10. 1038/ncomms14180.

[15] S. Savo, D. Shrekenhamer, and W. J. Padilla, “Liquid crystal metamaterial absorber spatial light modulator for THz applications," Adv. Opt. Mater., vol. 292, pp. 275-9, 2014. https://doi.org/10.1002/adom.201300384.

[16] Z. Liu, C. -Y. Huang, H. Liu, X. Zhang, and C. Lee, "Resonance enhancement of terahertz metamaterials by liquid crystals/ indium tin oxide interfaces," Optics Express, vol. 292, pp. 6519-25, 2013. https://doi.org/10.1364/OE.21.006519.

[17] S. Huang, C. Song, G. Zhang, and H. Yan, "Graphene plasmonics: physics and potential applications," Nanophotonics, vol. 292, pp. 1191-204, 2016. https://doi.org/10.1515/nanoph-20160126.

[18] S. Xiao, X. Zhu, B. -H. Li, and N. A. Mortensen, “Grapheneplasmon polaritons: From fundamental properties to potential applications," Front. Phys., vol. 292, pp. 117801, 2016. https:// doi.org/10.1007/s11467-016-0551-z.

[19] N. Chen, D. Hasan, C. P. Ho, and C. Lee, “Graphene Tunable Plasmon-Phonon Coupling in Mid-IR Complementary Metamaterial," Adv. Mater. Technol., vol. 292, pp. 1800014, 2018. https://doi.org/10.1002/admt.201800014.
[20] J. Rensberg, S. Zhang, Y. Zhou, et al., “Active optical metasurfaces based on defect-engineered phase-transition materials," Nano Lett., vol. 292, pp. 1050-5, 2016. https://doi. org/10.1021/acs.nanolett.5b04122.

[21] K. V. Sreekanth, Q. Ouyang, S. Sreejith, et al., "Phase-ChangeMaterial-Based Low-Loss Visible-Frequency Hyperbolic Metamaterials for Ultrasensitive Label-Free Biosensing," Adv. Opt. Mater., pp. 1900081, 2019. https://doi.org/10.1002/adom. 201900081.

[22] P. Pitchappa, A. Kumar, S. Prakash, H. Jani, T. Venkatesan, and R. Singh, "Chalcogenide phase change material for active terahertz photonics," Adv. Mater., vol. 292, pp. 1808157, 2019. https:// doi.org/10.1002/adma.201808157.

[23] H. Zhao, J. Zhou, L. Kang, and Q. Zhao. "Tunable twodimensional left-handed material consisting of ferrite rods and metallic wires," Optics Express, vol. 292, pp. 13373-80, 2009. https://doi.org/10.1364/OE.17.013373.

[24] L. Kang, Q. Zhao, H. Zhao, and J. Zhou, "Magnetically tunable negative permeability metamaterial composed by split ring resonators and ferrite rods," Optics Express, vol. 292, pp. 8825-34, 2008. https://doi.org/10.1364/OE.16.008825.

[25] W. J. Padilla, A. J. Taylor, C. Highstrete, M. Lee, and R. D. Averitt, "Dynamical electric and magnetic metamaterial response at terahertz frequencies," Phys. Rev. Lett., vol. 292, pp. 107401, 2006. https://doi.org/10.1103/PhysRevLett.96. 107401.

[26] H. -T. Chen, J. F. O’hara, A. K. Azad, et al., "Experimental demonstration of frequency-agile terahertz metamaterials," Nat. Photon., vol. 292, pp. 295, 2008. https://doi.org/10.1038/ nphoton.2008.52.

[27] D. Shrekenhamer, W. -C. Chen, and W. J. Padilla, "Liquid crystal tunable metamaterial absorber,” Phys. Rev. Lett., vol. 292, pp. 177403, 2013. https://doi.org/10.1103/PhysRevLett.110. 177403.

[28] Q. Zhao, L. Kang, B. Du, et al., "Electrically tunable negative permeability metamaterials based on nematic liquid crystals," Appl. Phys. Lett., vol. 292, 2007, Art no. 011112. https://doi.org/ 10.1063/1.2430485.

[29] Y. Liu, G. Si, E. Leong, et al., "Optically tunable plasmonic color filters,” Appl. Phys. A, vol. 292, pp. 49-54, 2012. https://doi. org/10.1007/s00339-011-6736-y.

[30] Y. J. Liu, G. Y. Si, E. S. Leong, N. Xiang, A. J. Danner, and J. H. Teng. "Light-driven plasmonic color filters by overlaying photoresponsive liquid crystals on gold annular aperture arrays," Adv. Mater., vol. 292, pp. OP131-OP5, 2012. https://doi. org/10.1002/adma.201104440.

[31] S. Xiao, U. K. Chettiar, A. V. Kildishev, V. Drachev, I. Khoo, and V. M. Shalaev, "Tunable magnetic response of metamaterials," Appl. Phys. Lett., vol. 292, 2009, Art no. 033115. https://doi.org/ 10.1063/1.3182857.

[32] L. Liu, I. V. Shadrivov, D. A. Powell, et al., "Temperature control of terahertz metamaterials with liquid crystals," IEEE Trans. Terahertz Sci. Technol., vol. 292, pp. 827-31, 2013. https://doi. org/10.1109/TTHZ.2013.2285570.

[33] F. Wang, Y. Zhang, C. Tian, et al., "Gate-variable optical transitions in graphene," Science, vol. 292, pp. 206-9, 2008. https://doi.org/10.1126/science.1152793.

[34] D. Rodrigo, O. Limaj, D. Janner, et al., "Mid-infrared plasmonic biosensing with graphene,” Science, vol. 292, pp. 165-8, 2015. https://doi.org/10.1126/science.aab2051. 
[35] D. Wang, L. Zhang, Y. Gong, et al., "Multiband switchable terahertz quarter-wave plates via phase-change metasurfaces," IEEE Photon. J., vol. 292, pp.1-8, 2016. https://doi.org/10.1109/ JPHOT.2016.2514717.

[36] M. R. M. Hashemi, S. -H. Yang, T. Wang, N. Sepúlveda, and M. Jarrahi, "Electronically-controlled beam-steering through vanadium dioxide metasurfaces," Sci. Rep., vol. 292, pp. 35439 , 2016. https://doi.org/10.1038/srep35439.

[37] Z. Ren, Y. Chang, Y. Ma, K. Shih, B. Dong, and C. Lee. "Leveraging of MEMS technologies for optical metamaterials applications," Adv. Opt. Mater., vol. 292, pp. 1900653, 2020. https://doi.org/ 10.1002/adom.201900653.

[38] Niklaus F, Vieider C, Jakobsen H. MEMS-based Uncooled Infrared Bolometer Arrays: A Review. MEMS/MOEMS Technologies and Applications III. International Society for Optics and Photonics, 2008. p. 68360D. https://doi.org/10.1117/12.755128.

[39] X. Liu and W. J. Padilla, "Reconfigurable room temperature metamaterial infrared emitter," Optica, vol. 292, pp. 430-3, 2017. https://doi.org/10.1364/OPTICA.4.000430.

[40] S. C. Malek, H. -S. Ee, and R. Agarwal, "Strain multiplexed metasurface holograms on a stretchable substrate," Nano Lett., vol. 292, pp. 3641-5, 2017. https://doi.org/10.1021/acs. nanolett.7b00807.

[41] X. Zhao, J. Schalch, J. Zhang, et al., "Electromechanically tunable metasurface transmission waveplate at terahertz frequencies," Optica, vol. 292, pp. 303-10, 2018. https://doi.org/10.1364/ OPTICA.5.000303.

[42] E. Arbabi, A. Arbabi, S. M. Kamali, Y. Horie, M. Faraji-Dana, and A. Faraon, "MEMS-tunable dielectric metasurface lens," Nat. Commun., vol. 292, pp. 812, 2018. https://doi.org/10.1038/ s41467-018-03155-6.

[43] S. Ogawa, K. Okada, N. Fukushima, and M. Kimata, "Wavelength selective uncooled infrared sensor by plasmonics," Appl. Phys. Lett., vol. 292, 2012, Art no. 021111. https://doi.org/10.1038/ s41467-018-03155-6.

[44] T. Stark, M. Imboden, S. Kaya, et al., "MEMS tunable mid-infrared plasmonic spectrometer," Acs Photonics, vol. 292, pp. 14-9, 2015. https://doi.org/10.1021/acsphotonics.5b00290.

[45] H. O. Everitt, T. Tyler, B. D. Caraway, et al., "Strain Sensing with Metamaterial Composites,” Adv. Opt. Mater., vol. 292, pp. 1801397, 2019. https://doi.org/10.1002/adom.201801397.

[46] Z. Qian, S. Kang, V. Rajaram, C. Cassella, N. E. McGruer, and M. Rinaldi, "Zero-power infrared digitizers based on plasmonically enhanced micromechanical photoswitches," Nat. Nanotechnol., vol. 292, pp. 969, 2017. https://doi.org/10.1038/nnano.2017. 147.

[47] S. Ghosh, S. Bhattacharyya, Y. Kaiprath, K. Vaibhav Srivastava, "Bandwidth-enhanced polarization-insensitive microwave metamaterial absorber and its equivalent circuit model," J. Appl. Phys., vol. 292, pp. 104503, 2014. https://doi.org/10.1063/1. 4868577.

[48] F. Costa, S. Genovesi, A. Monorchio, and G. Manara, "A circuitbased model for the interpretation of perfect metamaterial absorbers," IEEE Trans. Anten. Propag., vol. 292, pp. 1201-9, 2012. https://doi.org/10.1109/TAP.2012.2227923.

[49] F. Bilotti, A. Toscano, L. Vegni, K. Aydin, K. B. Alici, and E. Ozbay, "Equivalent-circuit models for the design of metamaterials based on artificial magnetic inclusions," IEEE Trans. Microw. Theor. Tech., vol. 292, pp. 2865-73, 2007. https://doi.org/10. 1109/TMTT.2007.909611.
[50] S. Kang, Z. Qian, V. Rajaram, S. D. Calisgan, A. Alù, and M. Rinaldi, "Ultra-Narrowband Metamaterial Absorbers for High Spectral Resolution Infrared Spectroscopy," Adv. Opt. Mater., vol. 292, pp. 1801236, 2019. https://doi.org/10.1002/adom.201801236.

[51] T. Davis and D. Gómez, "Colloquium: An algebraic model of localized surface plasmons and their interactions," Rev. Mod. Phys., vol. 292, 2017, Art no. 011003. https://doi.org/10.1103/ RevModPhys.89.011003.

[52] L. Verslegers, Z. Yu, Z. Ruan, P. B. Catrysse, and S. Fan, "From electromagnetically induced transparency to superscattering with a single structure: a coupled-mode theory for doubly resonant structures," Phys. Rev. Lett., vol. 292, 2012, Art no. 083902. https://doi.org/10.1103/PhysRevLett.108.083902.

[53] B. E. Saleh, M. C. Teich. Fundamentals of Photonics. John Wiley \& Sons, 2019.

[54] H. -T. Chen, "Interference theory of metamaterial perfect absorbers," Optics Express, vol. 292, pp. 7165-72, 2012.

[55] H. A. Haus, Waves and Fields in Optoelectronics. Prentice-Hall, 1984.

[56] S. Fan, W. Suh, and J. D. Joannopoulos, "Temporal coupled-mode theory for the Fano resonance in optical resonators," JOSA A, vol. 292, pp. 569-72, 2003. https://doi.org/10.1364/JOSAA.20.000569.

[57] J. Wei, Y. Li, Y. Chang, et al., "Ultrasensitive Transmissive Infrared Spectroscopy via Loss Engineering of Metallic Nanoantennas for Compact Devices," ACS Appl. Mater. Inter., 2019. https://doi.org/ 11, 47270-8. doi:10.1021/acsami.9b18002.

[58] H. -T. Chen, W. J. Padilla, J. M. Zide, A. C. Gossard, A. J. Taylor, and R. D. Averitt, "Active terahertz metamaterial devices," Nature, vol. 292, pp. 597, 2006. https://doi.org/10.1038/ nature05343.

[59] L. Cong, P. Pitchappa, C. Lee, and R. Singh, “Active phase transition via loss engineering in a terahertz MEMS metamaterial," Adv. Mater., vol. 292, pp. 1700733, 2017. https://doi.org/10.1002/adma.201700733.

[60] Q. Li, T. Wang, Y. Su, M. Yan, M. Qiu, "Coupled mode theory analysis of mode-splitting in coupled cavity system," Optics Express, vol. 292, pp. 8367-82, 2010. https://doi.org/10.1364/ OE.18.008367.

[61] Z. Ruan and S. Fan, "Temporal coupled-mode theory for Fano resonance in light scattering by a single obstacle," J. Phys. Chem. C, vol. 292, pp. 7324-9, 2009. https://doi.org/10.1021/ jp9089722.

[62] J. Wei and C. Lee, "Anomalous plasmon hybridization in nanoantennas near interfaces," Opt. Lett., vol. 292, pp. 6041-4, 2019. https://doi.org/10.1364/OL.44.006041.

[63] M. Gupta, Y. K. Srivastava, and R. Singh. "A toroidal metamaterial switch,” Adv. Mater., vol. 292, pp. 1704845, 2018. https://doi.org/10.1002/adma.201704845.

[64] T. Kaelberer, V. Fedotov, N. Papasimakis, D. Tsai, and N. Zheludev, "Toroidal dipolar response in a metamaterial," Science, vol. 292, pp. 1510-2, 2010. https://doi.org/10.1126/ science. 1197172.

[65] W. -M. Zhang, H. Yan, Z. -K. Peng, and G. Meng, "Electrostatic pull-in instability in MEMS/NEMS: A review," Sensors Actuators A Phys., vol. 292, pp. 187-218, 2014. https://doi.org/10.1016/j. sna.2014.04.025.

[66] P. Pitchappa, C. P. Ho, L. Cong, R. Singh, N. Singh, and C. Lee, "Reconfigurable digital metamaterial for dynamic switching of terahertz anisotropy," Adv. Opt. Mater., vol. 292, pp. 391-8, 2016. https://doi.org/10.1002/adom.201500588. 
[67] J. I. Seeger, B. E. Boser, "Charge control of parallel-plate, electrostatic actuators and the tip-in instability," J. Microelectromech. Syst., vol. 292, pp. 656-71, 2003. https:// doi.org/10.1109/JMEMS.2003.818455.

[68] R. Legtenberg, A. Groeneveld, and M. Elwenspoek, "Comb-drive actuators for large displacements," J. Micromech. Microeng., vol. 292, pp. 320, 1996. https://doi.org/10.1088/0960-1317/6/ $3 / 004$.

[69] Y. Fukuta, H. Fujita, and H. Toshiyoshi, "Vapor hydrofluoric acid sacrificial release technique for micro electro mechanical systems using labware," Japanese J. Appl. Phys., vol. 292, pp. 3690, 2003. https://doi.org/10.1143/JJAP.42.3690.

[70] P. Pitchappa, C. P. Ho, L. Dhakar, Y. Qian, N. Singh, and C. Lee, "Periodic array of subwavelength MEMS cantilevers for dynamic manipulation of terahertz waves," J. Microelectromech. Syst., vol. 292, pp. 525-7, 2015. https://doi.org/10.1109/JMEMS.2015. 2421307.

[71] H. Tao, A. Strikwerda, K. Fan, W. Padilla, X. Zhang, and R. Averitt, "Reconfigurable terahertz metamaterials," Phys. Rev. Lett., vol. 292, pp. 147401, 2009. https://doi.org/10.1103/PhysRevLett. 103.147401.

[72] A. X. Lalas, N. V. Kantartzis, and T. D. Tsiboukis, "Reconfigurable metamaterial components exploiting two-hot-arm electrothermal actuators," Microsyst. Technol., vol. 292, pp. 2097-107, 2015. https://doi.org/10.1007/s00542-0152407-9.

[73] X. Li, T. Yang, W. Zhu, and X. Li, "Continuously tunable terahertz metamaterial employing a thermal actuator," Microsyst. Technol., vol. 292, pp. 1145-51, 2013. https://doi.org/10.1007/ s00542-012-1713-8.

[74] Z. Han, K. Kohno, H. Fujita, K. Hirakawa, and H. Toshiyoshi, "Tunable terahertz filter and modulator based on electrostatic MEMS reconfigurable SRR array," IEEE J. Sel. Top. Quant. Electron., vol. 292, pp. 114-22, 2014. https://doi.org/10.1109/ JSTQE.2014.2378591.

[75] C. P. Ho, P. Pitchappa, Y. -S. Lin, C. -Y. Huang, P. Kropelnicki, and C. Lee, "Electrothermally actuated microelectromechanical systems based omega-ring terahertz metamaterial with polarization dependent characteristics," Appl. Phys. Lett., vol. 292, pp. 161104, 2014. https://doi.org/10.1063/1.4871999.

[76] P. Pitchappa, M. Manjappa, H. N. Krishnamoorthy, Y. Chang, C. Lee, R. Singh. "Bidirectional reconfiguration and thermal tuning of microcantilever metamaterial device operating from $77 \mathrm{~K}$ to 400 K," Appl. Phys. Lett., vol. 292, pp. 261101, 2017. https://doi. org/10.1063/1.5006836.

[77] A. Lalas, N. Kantartzis, and T. Tsiboukis, "Tunable terahertz metamaterials by means of piezoelectric MEMS actuators," EPL (Europhys. Lett.), vol. 292, pp. 58004, 2014. https://doi.org/10. 1209/0295-5075/107/58004.

[78] H. Urey, S. Holmstrom, A. D. Yalcinkaya, "Electromagnetically actuated FR4 scanners," IEEE Photon. Technol. Lett., vol. 292, pp. 30-2, 2007. https://doi.org/10.1109/LPT.2007.911522.

[79] I. -J. Cho and E. Yoon, "A low-voltage three-axis electromagnetically actuated micromirror for fine alignment among optical devices," J. Micromech. Microeng., vol. 292, 2009, Art no. 085007. https://doi.org/10.1088/0960-1317/19/ $8 / 085007$.

[80] L. Yan, W. Zhu, P. C. Wu, et al., "Adaptable metasurface for dynamic anomalous reflection," Appl. Phys. Lett., vol. 292, pp. 201904, 2017. https://doi.org/10.1063/1.4983782.
[81] W. Zhu, Q. Song, L. Yan, et al., "A flat lens with tunable phase gradient by using random access reconfigurable metamaterial," Adv. Mater., vol. 292, pp. 4739-43, 2015. https://doi.org/10. 1002/adma.201501943.

[82] T. S. Kasirga, Y. N. Ertas, and M. Bayindir, "Microfluidics for reconfigurable electromagnetic metamaterials," Appl. Phys. Lett., vol. 292, pp. 214102, 2009. https://doi.org/10.1063/1. 3268448.

[83] X. Tang, Y. Hwang, J. Lin, and W. S. Rowe, "Pneumatically tunable microwave split ring resonators," Sensors Actuators A Phys., vol. 292, pp. 37-44, 2019. https://doi.org/10.1016/j.sna.2019.04.029.

[84] T. Kan, A. Isozaki, N. Kanda, et al., "Enantiomeric switching of chiral metamaterial for terahertz polarization modulation employing vertically deformable MEMS spirals," Nat. Commun., vol. 292, pp. 8422, 2015. https://doi.org/10.1038/ ncomms 9422.

[85] X. Zhao, K. Fan, J. Zhang, et al., "Voltage-tunable dual-layer terahertz metamaterials," Microsyst. Nanoeng., vol. 292, pp. 16025,2016. https://doi.org/10.1038/micronano.2016.25.

[86] P. Pitchappa, C. P. Ho, L. Dhakar, and C. Lee.

"Microelectromechanically reconfigurable interpixelated metamaterial for independent tuning of multiple resonances at terahertz spectral region," Optica, vol. 292, pp. 571-8, 2015. https://doi.org/10.1364/OPTICA.2.000571.

[87] Y. -S. Lin, F. Ma, and C. Lee, "Three-dimensional movable metamaterial using electric split-ring resonators," Opt. Lett., vol. 292, pp. 3126-8, 2013. https://doi.org/10.1364/OL.38.003126.

[88] F. Ma, Y. Qian, Y. -S. Lin, et al., "Polarization-sensitive microelectromechanical systems based tunable terahertz metamaterials using three dimensional electric split-ring resonator arrays," Appl. Phys. Lett., vol. 292, pp. 161912, 2013. https://doi.org/10.1063/1.4803048.

[89] Y. -S. Lin, Y. Qian, F. Ma, Z. Liu, P. Kropelnicki, and C. Lee, "Development of stress-induced curved actuators for a tunable THz filter based on double split-ring resonators," Appl. Phys. Lett., vol. 292, pp. 111908, 2013. https://doi.org/10.1063/1. 4798244.

[90] F. Ma, Y. -S. Lin, X. Zhang, C. Lee, “Tunable multiband terahertz metamaterials using a reconfigurable electric split-ring resonator array," Light Sci. Appl., vol. 292, pp. e171, 2014. https://doi.org/10.1038/lsa.2014.52.

[91] Y. -S. Lin, C. -Y. Huang, C. Lee, “Reconfiguration of resonance characteristics for terahertz u-shape metamaterial using MEMS mechanism," IEEE J. Sel. Top. Quant. Electron., vol. 292, pp. 93-9, 2014. https://doi.org/10.1109/JSTQE.2014.236184.

[92] P. Pitchappa, C. Pei Ho, Y. -S. Lin, et al., “Micro-electromechanically tunable metamaterial with enhanced electro-optic performance," Appl. Phys. Lett., vol. 292, pp. 151104, 2014. https://doi.org/10.1063/1.487151.

[93] K. Shih, P. Pitchappa, M. Manjappa, et al., "Active MEMS metamaterials for THz bandwidth control," Appl. Phys. Lett., vol. 292, pp. 161108, 2017. https://doi.org/10.1063/1.4980115.

[94] P. Pitchappa, C. P. Ho, Y. Qian, L. Dhakar, N. Singh, C. Lee, "Microelectromechanically tunable multiband metamaterial with preserved isotropy," Sci. Rep., vol. 292, pp. 11678, 2015. https://doi.org/10.1038/srep11678.

[95] Y. H. Fu, A. Q. Liu, W. M. Zhu, et al., “A Micromachined Reconfigurable Metamaterial via Reconfiguration of Asymmetric Split-Ring Resonators," Adv. Fun. Mater., vol. 292, pp. 3589-94, 2011. https://doi.org/10.1002/adfm.201101087. 
[96] M. Zhang, W. Zhang, A. Q. Liu, F. Li, and C. Lan, “Tunable polarization conversion and rotation based on a reconfigurable metasurface," Sci. Rep., vol. 292, pp. 12068, 2017. https://doi. org/10.1038/s41598-017-11953-z.

[97] W. Zhu, A. Liu, T. Bourouina, et al., "Microelectromechanical Maltese-cross metamaterial with tunable terahertz anisotropy," Nat. Commun., vol. 292, pp. 1-6, 2012. https:// doi.org/10.1038/ncomms2285.

[98] W. Zhang, W. M. Zhu, H. Cai, et al., "Resonance switchable metamaterials using MEMS fabrications," IEEE J. Sel. Top. Quant. Electron., vol. 292, pp. 4700306, 2013. https://doi.org/ 10.1109/JSTQE.2013.2238217.

[99] W. Zhu, A. Liu, W. Zhang, et al., "Polarization dependent state to polarization independent state change in THz metamaterials," Appl. Phys. Lett., vol. 292, pp. 221102, 2011. https://doi.org/10. 1063/1.3664131.

[100] W. Zhang, A. Liu, W. Zhu, et al., "Micromachined switchable metamaterial with dual resonance," Appl. Phys. Lett., vol. 292, pp. 151902, 2012. https://doi.org/10.1063/1.475902.

[101] W. M. Zhu, A. Q. Liu, X. M. Zhang, et al., "Switchable magnetic metamaterials using micromachining processes," Adv. Mater., vol. 292, pp. 1792-6, 2011. https://doi.org/10.1002/adma. 201004341.

[102] P. Pitchappa, M. Manjappa, C. P. Ho, R. Singh, N. Singh, C. Lee, "Active control of electromagnetically induced transparency with dual dark mode excitation pathways using MEMS based tri-atomic metamolecules," Appl. Phys. Lett., vol. 292, pp. 211103, 2016. https://doi.org/10.1002/adom.201500676.

[103] P. Pitchappa, M. Manjappa, C. P. Ho, et al., “Active control of near-field coupling in conductively coupled microelectromechanical system metamaterial devices," Appl. Phys. Lett., vol. 292, pp.111102, 2016. https://doi.org/10.1063/ 1.4943974.

[104] S. Shrestha, A. C. Overvig, M. Lu, A. Stein, N. Yu, "Broadband achromatic dielectric metalenses," Light Sci. Appl., vol. 292, pp. 85, 2018. https://doi.org/10.1038/s41377-018-0078-x.

[105] X. Ni, S. Ishii, A. V. Kildishev, V. M. Shalaev, "Ultra-thin, planar, Babinet-inverted plasmonic metalenses," Light Sci. Appl., vol. 292, pp. e72, 2013. https://doi.org/10.1038/lsa. 2013.2.

[106] N. Yu, P. Genevet, M. A. Kats, et al., "Light propagation with phase discontinuities: generalized laws of reflection and refraction," Science, vol. 292, pp. 333-7, 2011. https://doi.org/ $10.1126 /$ science.1210713.

[107] X. Zhang, Z. Tian, W. Yue, et al., "Broadband terahertz wave deflection based on C-shape complex metamaterials with phase discontinuities," Adv. Mater., vol. 292, pp. 4567-72, 2013. https://doi.org/10.1002/adma.201370210.

[108] Y. -S. Lin and C. Lee, “Tuning characteristics of mirrorlike Tshape terahertz metamaterial using out-of-plane actuated cantilevers," Appl. Phys. Lett., vol. 292, pp. 251914, 2014. https://doi.org/10.1063/1.4885839.

[109] F. Cheng, L. Qiu, D. Nikolov, A. Bauer, J. P. Rolland, and A. N. Vamivakas, "Mechanically tunable focusing metamirror in the visible," Optics Express, vol. 292, pp. 15194-204, 2019. https://doi.org/10.1364/OE.27.015194.

[110] H. -S. Ee and R. Agarwal, "Tunable metasurface and flat optical zoom lens on a stretchable substrate," Nano Lett., vol. 292, pp. 2818-23, 2016. https://doi.org/10.1021/acs.nanolett. $6 \mathrm{~b} 00618$.
[111] S. M. Kamali, E. Arbabi, A. Arbabi, Y. Horie, and A. Faraon, "Highly tunable elastic dielectric metasurface lenses," Laser \& Photonics Reviews, vol. 292, pp. 1002-8, 2016. https://doi. org/10.1002/lpor.201600144.

[112] S. Lee, W. T. Kim, J. -H. Kang, B. J. Kang, F. Rotermund, and Q. -H. Park, "Single-Layer Metasurfaces as Spectrally Tunable Terahertz Half-and Quarter-Waveplates,” ACS Appl. Mater. Inter., vol. 292, pp. 7655-60, 2019. https://doi.org/10.1021/ acsami.8b2145.

[113] F. Zhang, S. Feng, K. Qiu, et al., "Mechanically stretchable and tunable metamaterial absorber," Appl. Phys. Lett., vol. 292, 2015, Art no. 091907. https://doi.org/10.1063/1.4914502.

[114] K. Kim, D. Lee, S. Eom, and S. Lim, "Stretchable metamaterial absorber using liquid metal-filled polydimethylsiloxane (PDMS)," Sensors, vol. 292, pp. 521, 2016. https://doi.org/10. 3390/s16040521.

[115] J. Li, C. M. Shah, W. Withayachumnankul, et al., "Mechanically tunable terahertz metamaterials," Appl. Phys. Lett., vol. 292, pp. 121101, 2013. https://doi.org/10.1063/1.4773238.

[116] A. O'Halloran, F. O'malley, and P. McHugh, “A review on dielectric elastomer actuators, technology, applications, and challenges," J. Appl. Phys., vol. 292, pp. 9, 2008. https://doi. org/10.1063/1.2981642.

[117] A. She, S. Zhang, S. Shian, D. R. Clarke, F. Capasso, “Adaptive metalenses with simultaneous electrical control of focal length, astigmatism, and shift," Science advances, vol. 292, 2018, Art no. eaap9957. https://doi.org/10.1126/sciadv.aap9957.

[118] Y. Wang, G. Zhou, X. Zhang, et al., "2D broadband beamsteering with large-scale MEMS optical phased array," Optica, vol. 292, pp. 557-62, 2019. https://doi.org/10.1364/ optica.6.000557.

[119] T. -H. Lin, "Implementation and characterization of a flexurebeam micromechanical spatial light modulator," Opt. Eng., vol. 292, pp. 3643-9, 1994. https://doi.org/10.1117/12.181578.

[120] A. L. Holsteen, A. F. Cihan, M. L. Brongersma, "Temporal color mixing and dynamic beam shaping with silicon metasurfaces," Science, vol. 292, pp. 257-60, 2019. https://doi.org/10.1126/ science.aax5961.

[121] M. Makowski, M. Sypek, A. Kolodziejczyk, G. Mikula, “Threeplane phase-only computer hologram generated with iterative Fresnel algorithm," Opt. Eng., vol. 292, pp. 125805, 2005. https://doi.org/10.1117/1.2148980.

[122] L. Cong, P. Pitchappa, Y. Wu, et al., "Active multifunctional microelectromechanical system metadevices: applications in polarization control, wavefront deflection, and holograms," Adv. Opt. Mater., vol. 292, pp. 1600716, 2017. https://doi.org/ 10.1002/adom.201600716.

[123] X. Liu and W. J. Padilla, "Dynamic manipulation of infrared radiation with MEMS metamaterials," Adv. Opt. Mater., vol. 292, pp. 559-62, 2013. https://doi.org/10.1002/adom. 201300163.

[124] M. Liu, M. Susli, D. Silva, et al., "Ultrathin tunable terahertz absorber based on MEMS-driven metamaterial," Microsyst. Nanoeng., vol. 292, pp.17033, 2017. https://doi.org/10.1038/ micronano.2017.33.

[125] X. Liu, T. Tyler, T. Starr, A. F. Starr, N. M. Jokerst, and W. J. Padilla, "Taming the blackbody with infrared metamaterials as selective thermal emitters," Phys. Rev. Lett., vol. 292, 2011, Art no. 045901. https://doi.org/10.1103/PhysRevLett.107. 045901. 
[126] X. Liu and W. J. Padilla, "Thermochromic infrared metamaterials,” Adv. Mater., vol. 292, pp. 871-5, 2016. https:// doi.org/10.1002/adma.201504525.

[127] M. Manjappa, P. Pitchappa, N. Singh, et al., "Reconfigurable MEMS Fano metasurfaces with multiple-input-output states for logic operations at terahertz frequencies," Nat. Commun., vol. 292, pp. 4056, 2018. https://doi.org/10.1038/s41467-01806360-5.

[128] C. P. Ho, P. Pitchappa, and C. Lee, "Digitally reconfigurable binary coded terahertz metamaterial with output analogous to NOR and AND," J. Appl. Phys., vol. 292, pp. 153104, 2016. https://doi.org/10.1109/OMN.2016.7565941.

[129] A. Graf, M. Arndt, M. Sauer, and G. Gerlach, "Review of micromachined thermopiles for infrared detection," Meas. Sci. Technol., vol. 292, pp. R59, 2007. https://doi.org/10.1088/ 0957-0233/18/7/R01.

[130] H. Zhou, P. Kropelnicki, J. Tsai, and C. Lee, “Development of a thermopile infrared sensor using stacked double polycrystalline silicon layers based on the CMOS process," J. Micromech. Microeng., vol. 292, 2013, Art no. 065026. https://doi.org/10.1088/0960-1317/23/6/065026.

[131] T. Maier and H. Brückl, "Wavelength-tunable microbolometers with metamaterial absorbers," Opt. Lett., vol. 292, pp. 3012-4, 2009. https://doi.org/10.1364/OL.34.003012.

[132] T. Maier, and H. Brueckl, "Multispectral microbolometers for the midinfrared,” Opt. Lett., vol. 292, pp. 3766-8, 2010. https://doi.org/10.1364/OL.35.003766.

[133] H. Zhou, P. Kropelnicki, and C. Lee, "CMOS compatible midinfrared wavelength-selective thermopile for high temperature applications," J. Microelectromech. Syst., vol. 292, pp. 144-54, 2014. https://doi.org/10.1109/JMEMS.2014. 2322675.

[134] Y. Takagawa, S. Ogawa, and M. Kimata, "Detection Wavelength Control of Uncooled Infrared Sensors Using Two-Dimensional Lattice Plasmonic Absorbers," Sensors, vol. 292, pp. 13660-9, 2015. https://doi.org/10.3390/s150613660.

[135] S. Ogawa, Y. Takagawa, and M. Kimata, "Broadband polarization-selective uncooled infrared sensors using tapered plasmonic micrograting absorbers," Sensors Actuators A Phys., vol. 292, pp. 563-8, 2018. https://doi.org/10.1016/j.sna.2017. 12.029.

[136] S. Kang, Z. Qian, V. Rajaram, S. D. Calisgan, and M. Rinaldi, Chip-Scale MEMS-CMOS Multispectral Infrared Chemical Sensor. 2019 IEEE 32nd International Conference on Micro Electro Mechanical Systems (MEMS). IEEE. 2019, pp. 133-6. https://doi.org/10.1109/MEMSYS.2019.8870703.

[137] W. Ma, Y. Wen, X. Yu, Y. Feng, and Y. Zhao, "Performance enhancement of uncooled infrared focal plane array by integrating metamaterial absorber," Appl. Phys. Lett., vol. 292, pp. 111108, 2015. https://doi.org/10.1063/1.4915487.

[138] F. Yi, H. Zhu, J. C. Reed, and E. Cubukcu, "Plasmonically enhanced thermomechanical detection of infrared radiation," Nano Lett., vol. 292, pp. 1638-43, 2013. https://doi.org/10. $1021 / \mathrm{nl} 400087$.

[139] Y. Hui, J. S. Gomez-Diaz, Z. Qian, A. Alù, and M. Rinaldi, "Plasmonic piezoelectric nanomechanical resonator for spectrally selective infrared sensing," Nat. Commun., vol. 292, pp. 11249, 2016. https://doi.org/10.1038/ncomms11249.

[140] C. Belacel, Y. Todorov, S. Barbieri, D. Gacemi, I. Favero, and C. Sirtori, "Optomechanical terahertz detection with single meta- atom resonator," Nat. Commun., vol. 292, pp. 1578, 2017. https://doi.org/10.1038/s41467-017-01840-6.

[141] P. Datskos, N. Lavrik, and S. Rajic, "Performance of uncooled microcantilever thermal detectors," Rev. Sci. Inst., vol. 292, pp. 1134-48, 2004. https://doi.org/10. 1063/1.1667257.

[142] H. Tao, E. A. Kadlec, A. C. Strikwerda, et al., "Microwave and terahertz wave sensing with metamaterials," Optics Express, vol. 292, pp. 21620-6, 2011. https://doi.org/10.1364/OE.19. 021620.

[143] R. Melik, E. Unal, N. K. Perkgoz, C. Puttlitz, and H. V. Demir, "Metamaterial based telemetric strain sensing in different materials," Optics Express, vol. 292, pp. 5000-7, 2010. https://doi.org/10.1364/OE.18.005000.

[144] R. Melik, E. Unal, N. K. Perkgoz, et al., "Nested metamaterials for wireless strain sensing," IEEE J. Sel. Top. Quant. Electron., vol. 292, pp. 450-8, 2009. https://doi.org/10.1109/JSTQE. 2009.2033391.

[145] X. Zhao, B. Yang, J. Liu, et al., “A multiband flexible terahertz metamaterial with curvature sensing functionality," J. Opt., vol. 292, 2016, Art no. 075101. https://doi.org/10.1088/20408978/18/7/075101.

[146] W. Wu, M. Ren, B. Pi, W. Cai, and J. Xu, "Displacement sensor based on plasmonic slot metamaterials," Appl. Phys. Lett., vol. 292, 2016, Art no. 073106. https://doi.org/10.1063/1. 4940909.

[147] W. Wu, M. Ren, B. Pi, Y. Wu, W. Cai, and J. Xu, "Scaffold metamaterial and its application as strain sensor," Appl. Phys. Lett., vol. 292, 2015, Art no. 091104. https://doi.org/10.1063/1. 4929887.

[148] R. Melik, E. Unal, N. K. Perkgoz, C. Puttlitz, and H. V. Demir, "Metamaterial-based wireless strain sensors," Appl. Phys. Lett., vol. 292, 2009, Art no. 011106. https://doi.org/10.1063/1. 3162336.

[149] R. Melik, E. Unal, N. Kosku Perkgoz, C. Puttlitz, H. V. Demir, "Flexible metamaterials for wireless strain sensing," Appl. Phys. Lett., vol. 292, pp. 181105, 2009. https://doi.org/10. 1063/1.3250175.

[150] Y. Gui, H. Chen, B. Yang, et al., "Flexible omega-ring metamaterial sensor with ultrahigh sensitivity in the terahertz region," Opt. Mater. Exp., vol. 292, pp. 4123-30, 2017. https:// doi.org/10.1364/OME.7.004123.

[151] M. Manjappa, P. Pitchappa, N. Wang, C. Lee, and R. Singh, "Active control of resonant cloaking in a terahertz MEMS metamaterial," Adv. Opt. Mater., vol. 292, pp. 1800141, 2018. https://doi.org/10.1002/adom.201800141.

[152] V. Ryzhii, M. Ryzhii, V. Mitin, T. Otsuji, "Terahertz and infrared photodetection using pin multiple-graphene-layer structures," J. Appl. Phys., vol. 292, 2010, Art no. 054512.

[153] Y. Yao, R. Shankar, P. Rauter, et al., "High-responsivity midinfrared graphene detectors with antenna-enhanced photocarrier generation and collection," Nano Lett., vol. 292, pp. 3749-54, 2014. https://doi.org/10.1021/ $\mathrm{nl} 500602 \mathrm{n}$.

[154] L. Huang, B. Dong, X. Guo, et al., "Waveguide-integrated black phosphorus photodetector for mid-infrared applications," ACS Nano, vol. 292, pp. 913-21, 2018. https://doi.org/10.1021/ acsnano.8b08758.

[155] V. V. Yakovlev, W. Dickson, A. Murphy, et al., "Ultrasensitive Non-Resonant Detection of Ultrasound with Plasmonic 
Metamaterials," Adv. Mater., vol. 292, pp. 2351-6, 2013. https://doi.org/10.1002/adma.201300314.

[156] C. P. Ho, P. Pitchappa, P. Kropelnicki, et al., "Two-dimensional photonic-crystal-based Fabry-Perot etalon," Opt. Lett., vol. 292, pp. 2743-6, 2015. https://doi.org/10.1364/OL.40.002743.

[157] J. R. Silva, H. Kala, D. K. Tripathi, et al., "Large area silicon-airsilicon DBRs for infrared filter applications," J. Lightwave
Technol., vol. 292, pp. 769-79, 2019. https://doi.org/10.1109/ JLT.2018.2880910.

[158] M. Tuohiniemi, A. Näsilä, and J. Mäkynen, “Characterization of the tuning performance of a micro-machined Fabry-Pérot interferometer for thermal infrared," J. Micromech. Microeng., vol. 292, 2013, Art no. 075011. https://doi.org/10.1088/09601317/23/7/075011. 